\title{
How Non-Gaussian Shocks Affect Risk Premia in Non- Linear DSGE Models
}

\author{
Martin M. Andreasen
}

School of Economics and Management

Aarhus University

Bartholins Allé 10, Building 1322, DK-8000 Aarhus C

Denmark 


\title{
How Non-Gaussian Shocks Affect Risk Premia in Non-Linear DSGE Models
}

\author{
Martin M. Andreasen* \\ Bank of England and CREATES
}

September 10, 2010

\begin{abstract}
This paper studies how non-Gaussian shocks affect risk premia in DSGE models approximated to second and third order. Based on an extension of the results in Schmitt-Grohé \& Uribe (2004) to third order, we derive propositions for how rare disasters, stochastic volatility, and GARCH affect any risk premia in a wide class of DSGE models. To quantify these effects, we then set up a standard New Keynesian DSGE model where total factor productivity includes rare disasters, stochastic volatility, and GARCH. We find that rare disasters increase the mean level of the 10-year nominal term premium, whereas a key effect of stochastic volatility and GARCH is an increase in the variability of this premium.

Keywords: Epstain-Zin-Weil preferences, GARCH, rare disasters, risk premia, stochastic volatility.
\end{abstract}

JEL: C68, E30, E43, E44

${ }^{*}$ I would like to thank Juan Rubio-Ramírez for useful comments and discussions. A special thank to Eric Swanson for providing US data on the 10-year interest rate. I greatly acknowledge assess to computer facilities as provided by the Danish Center for Scientific Computation (DCSC). I also appreciate financial support to Center for Research in Econometric Analysis of Time Series, CREATES, funded by the Danish National Research Foundation. Finally, the views expressed herein are solely those of the author and not necessarily those of the Bank of England. Email: martin.andreasen@bankofengland.co.uk. Telephone number: +44 2076013431. 


\section{Introduction}

Macroeconomic models often struggle to explain the dynamics of asset prices and their related risk premia. The early work by Mehra \& Prescott (1985), Mark (1985), and Backus, Gregory \& Zin (1989) illustrate this for the equity premium, the foreign exchange risk premium, and the term premium, respectively. Much work have subsequently tried to improve the standard consumption endowment model along these dimensions. The paper by Campbell \& Cochrane (1999) extend the model with consumption habits and heteroscedastic shocks, whereas Bansal \& Yaron (2004) emphasize the importance of Epstein-Zin-Weil preferences and stochastic volatility in consumption growth. Another interesting extension is due to Rietz (1988) and Barro (2006) who also rely on non-Gaussian shocks as they incorporate rare disasters into the basic framework. These improvements of the consumption endowment model are all successful at reproducing several key moments of asset prices.

A second strand of the literature tries to explain the same asset pricing moments in fully specified Dynamic Stochastic General Equilibrium (DSGE) models. The objective in these models is to provide further economic insight into the dynamics of asset prices through general equilibrium effects. Important contributions are Jermann (1998), Boldrin, Christiano \& Fisher (2001), Chari, Kehoe \& McGrattan (2002) and more recently Wu (2006), Uhlig (2007), De Paoli, Scott \& Weeken (2007), Hordahl, Tristani \& Vestin (2008), Rudebusch \& Swanson (2009), and Bekaert, Cho \& Moreno (2010). It is interesting to note that all these studies only consider Gaussian shocks when specifying structural disturbances. This is contrary to the aforementioned literature based on endowment economies which rely heavily on non-Gaussian shocks. Moreover, the exclusive focus on Gaussian shocks when studying asset prices in DSGE models also goes counter to recent findings suggesting that structural innovations may be non-Gaussian (see Fernández-Villaverde \& Rubio-Ramírez (2007), Justiniano \& Primiceri (2008), Bloom (2009), Fernández-Villaverde, Guerrón-Quintana, Rubio-Ramírez \& Uribe (2010), among others).

The contribution of this paper is to close this gap in the literature and study how nonGaussian shocks in DSGE models affect risk premia. This is done for the general class of DSGE models considered in Schmitt-Grohé \& Uribe (2004) when these models are solved by second and third order perturbation approximations around the deterministic steady state.

The third order terms are of great economic interest because they allow for time-varying risk premia as observed in the data (see for instance Campbell \& Shiller (1991)). Schmitt-Grohé \& Uribe (2004) derive the solution to all second order terms and we extend their results to third order. These analytical formulas are useful because they provide key insights into how non-Gaussian shocks affect the approximated solution. Similar insights are hard to establish when approximations to DSGE models are computed in a high-level programming language like Mathematica (see Swanson, Anderson \& Levin (2005), Arouba, Fernández-Villaverde \& Rubio-Ramírez (2005), Rudebusch \& Swanson (2009), among others). 
Based on the general formulas for second and third order terms in DSGE models, we then analyze how non-Gaussian shocks in general affect risk premia in these models. Given the aforementioned extensions of the standard endowment model, we choose to explore effects of rare disasters, stochastic volatility, and conditional heteroscedasticity modelled by GARCH. Our key findings are as follows. Firstly, the presence of rare disasters does not affect risk premia in a second order approximation and only change the level of risk premia at third order. Secondly, modelling time-varying uncertainty by stochastic volatility and GARCH do not generate timevarying risk premia in a second order approximation. This is because all second moments remain constant at the approximation point even with these extensions. Thirdly, when DSGE models are solved up to third order, stochastic volatility and GARCH may affect the level of risk premia, and these processes may generate additional variation in risk premia. We emphasize that these properties hold for all DSGE models belonging to the considered class and for any risk premia - i.e. whether it relates to equities, bonds, exchange rates, etc.

To explore the quantitative effects of non-Gaussian shocks, we then examine how rare disasters, stochastic volatility, and GARCH in productivity shocks affect the 10-year nominal term premium in an otherwise standard New Keynesian DSGE model solved to third order. We find that the chosen specification of rare disasters can have substantial effects on the level of the term premium and values of skewness and kurtosis for several macro variables. However, rare disasters hardly affect the standard deviation of most macro variables. We also find that stochastic volatility can generate sizeable variation in the term premium without distorting the model's ability to match a key number of macro economic moments. A decomposition shows that stochastic volatility makes this premium more volatile by increasing the variation in the quantity of risk. Hence, our DSGE model has the same key feature as many finance models; a higher uncertainty level raises the term premium through an increase in the quantity of risk (see Cox, Ingersoll \& Ross (1985), Dai \& Singleton (2002), among others). We also find that the considered specification of stochastic volatility does not change the mean level of the term premium. The effects of GARCH are slightly different from those generated by stochastic volatility. In particular, GARCH increases both the mean level and the variability of the term premium. A decomposition shows that these effects arise from a higher and more volatile market price of risk. It is further shown that GARCH affects the variability of consumption growth but not the variation in inflation and interest rates.

From our analysis we therefore conclude that non-Gaussian shocks can have substantial effects on risk premia in DSGE models. In particular, non-Gaussian shocks may generate more realistic and less puzzling dynamics for risk premia without compromising the ability of the models to match other moments.

The rest of this paper is organized as follows. Section 2 introduces the setup considered in Schmitt-Grohé \& Uribe (2004), and section 3 derives analytical expressions for all third order terms in this class of DSGE models. The general expression for risk premia is studied in section 
4 where we also analyze the effects of innovations with non-symmetric distributions (i.e. rare disasters), stochastic volatility, and GARCH for risk premia. A standard New Keynesian model is considered in section 5 to explore the quantitative effects of these shock specifications for the 10-year nominal term premium. Concluding comments are provided in section 6 . Unless stated otherwise, all proofs are deferred to a technical appendix available from the author's web page or on request.

\section{The general model}

Following Schmitt-Grohé \& Uribe (2004), we consider models with equilibrium conditions of the form

$$
E_{t}\left[\mathbf{f}\left(\mathbf{y}_{t+1}, \mathbf{y}_{t}, \mathbf{x}_{t+1}, \mathbf{x}_{t}\right)\right]=\mathbf{0},
$$

where $E_{t}$ is the conditional expectation given information available at period $t$. The state vector $\mathbf{x}_{t}$ has dimension $n_{x} \times 1$, and the vector $\mathbf{y}_{t}$ with dimension $n_{y} \times 1$ contains all the control variables. Here, $n_{x}+n_{y} \equiv n$. The function $\mathbf{f}$ takes elements from $\mathbb{R}^{n_{y}} \times \mathbb{R}^{n_{y}} \times \mathbb{R}^{n_{x}} \times \mathbb{R}^{n_{x}}$ into $\mathbb{R}^{n}$, and we assume that this mapping is at least three times differentiable in the deterministic steady state. This point is defined by $\sigma=0$ and values of $\left(\mathbf{x}_{s s}, \mathbf{y}_{s s}\right)$ which imply $\mathbf{f}\left(\mathbf{y}_{s s}, \mathbf{y}_{s s}, \mathbf{x}_{s s}, \mathbf{x}_{s s}\right)=\mathbf{0}$.

The vector $\mathbf{x}_{t}$ is partitioned as $\left[\begin{array}{ll}\mathbf{x}_{1, t}^{\prime} & \mathbf{x}_{2, t}^{\prime}\end{array}\right]^{\prime}$ where $\mathbf{x}_{1, t}$ contains endogenous state variables and $\mathbf{x}_{2, t}$ contains exogenous state variables. The dimensions of these vectors are $n_{x_{1}} \times 1$ and $n_{x_{2}} \times 1$, respectively, where $n_{x_{1}}+n_{x_{2}}=n_{x}$. It is further assumed that

$$
\mathbf{x}_{t}^{2}=\boldsymbol{\Gamma}\left(\mathbf{x}_{t}^{2}\right)+\sigma \tilde{\boldsymbol{\eta}} \boldsymbol{\epsilon}_{t+1}
$$

where $\boldsymbol{\epsilon}_{t+1}$ has dimension $n_{\epsilon} \times 1$ and is independent and identical distributed with mean zero and covariance matrix $\mathbf{I}$. That is, $\boldsymbol{\epsilon}_{t+1} \sim \mathcal{I} \mathcal{I D}(\mathbf{0}, \mathbf{I})$. The function $\boldsymbol{\Gamma}$ takes elements from $\mathbb{R}^{n_{x_{2}}}$ into $\mathbb{R}^{n_{x_{2}}}$ and is required to be at least three times differentiable in the deterministic steady state. Moreover, all eigenvalues of $\partial \boldsymbol{\Gamma} / \partial \mathbf{x}_{2, t}$ evaluated in the deterministic steady state must have modulus less than one. Finally, $\sigma \geq 0$ and $\tilde{\boldsymbol{\eta}}$ is a known matrix with dimension $n_{x_{2}} \times n_{\epsilon}$

As observed by Schmitt-Grohé \& Uribe (2004), the solution to this model is given by

$$
\begin{gathered}
\mathbf{y}_{t}=\mathbf{g}\left(\mathbf{x}_{t}, \sigma\right) \\
\mathbf{x}_{t+1}=\mathbf{h}\left(\mathbf{x}_{t}, \sigma\right)+\sigma \boldsymbol{\eta} \boldsymbol{\epsilon}_{t+1} \\
\boldsymbol{\eta} \equiv\left[\begin{array}{c}
\mathbf{0}_{n_{x_{1}} \times n_{\epsilon}} \\
\tilde{\boldsymbol{\eta}}
\end{array}\right]
\end{gathered}
$$

The function $\mathbf{g}$ maps elements from $\mathbb{R}^{n_{x}} \times \mathbb{R}^{+}$into $\mathbb{R}^{n_{y}}$ whereas $\mathbf{h}$ takes elements from $\mathbb{R}^{n_{x}} \times \mathbb{R}^{+}$ into $\mathbb{R}^{n_{x}}$. Both functions are unknown and assumed to be at least three times continuous 
differentiable at the deterministic steady state.

\section{A third order approximation}

Substituting (3)-(4) into (1) gives

$$
\mathbf{F}(\mathbf{x}, \sigma) \equiv E_{t}\left[\mathbf{f}\left(\mathbf{g}\left(\mathbf{h}(\mathbf{x}, \sigma)+\sigma \boldsymbol{\eta} \boldsymbol{\epsilon}^{\prime}, \sigma\right), \mathbf{g}(\mathbf{x}, \sigma), \mathbf{h}(\mathbf{x}, \sigma)+\sigma \boldsymbol{\eta} \boldsymbol{\epsilon}^{\prime}, \mathbf{x}\right)\right]=\mathbf{0}
$$

For simplicity, we omit the time subscript and use a prime to denote variables in period $t+1$. A third order approximation to $\mathbf{g}$ and $\mathbf{h}$ is stated in the appendix. The first and second order derivatives of $\mathbf{g}$ and $\mathbf{h}$ at the deterministic steady state are computed in Schmitt-Grohé \& Uribe (2004). We now derive the expression for all third order terms using the fact that derivatives of $\mathbf{F}(\mathbf{x}, \sigma)$ are zero. Computing these third order terms is greatly simplified by the fact that only linear systems need to be solved as noted by Judd \& Guu (1997).

\subsection{Computing the third order terms}

We start by computing $\mathbf{g}_{\mathbf{x} \mathbf{x} \mathbf{x}}$ and $\mathbf{h}_{\mathbf{x} \mathbf{x} \mathbf{x}}$ which is done from the third derivative of $\mathbf{F}(\mathbf{x}, \sigma)$ with respect to $\mathbf{x}$ and evaluated in the deterministic steady state. This gives

$$
\begin{aligned}
{\left[\mathbf{F}_{\mathbf{x x x}}\left(\mathbf{x}_{s s}, \sigma\right)\right]_{\alpha_{1} \alpha_{2} \alpha_{3}}^{i}=} & {\left[\mathbf{f}_{\mathbf{y}^{\prime}}\right]_{\beta_{1}}^{i}\left[\mathbf{g}_{\mathbf{x x x}}\right]_{\gamma_{1} \gamma_{2} \gamma_{3}}^{\beta_{1}}\left[\mathbf{h}_{\mathbf{x}}\right]_{\alpha_{3}}^{\gamma_{3}}\left[\mathbf{h}_{\mathbf{x}}\right]_{\alpha_{2}}^{\gamma_{2}}\left[\mathbf{h}_{\mathbf{x}}\right]_{\alpha_{1}}^{\gamma_{1}}+\left[\mathbf{f}_{\mathbf{y}^{\prime}}\right]_{\beta_{1}}^{i}\left[\mathbf{g}_{\mathbf{x}}\right]_{\gamma_{1}}^{\beta_{1}}\left[\mathbf{h}_{\mathbf{x x x}}\right]_{\alpha_{1} \alpha_{2} \alpha_{3}}^{\gamma_{1}}(7) } \\
& +\left[\mathbf{f}_{\mathbf{y}}\right]_{\beta_{1}}^{i}\left[\mathbf{g}_{\mathbf{x x x}}\right]_{\alpha_{1} \alpha_{2} \alpha_{3}}^{\beta_{1}}+\left[\mathbf{f}_{\mathbf{x}^{\prime}}\right]_{\gamma_{1}}^{i}\left[\mathbf{h}_{\mathbf{x x x}}\right]_{\alpha_{1} \alpha_{2} \alpha_{3}}^{\gamma_{1}}+\left[b^{1}\right]_{\alpha_{1} \alpha_{2} \alpha_{3}}^{i} \\
= & 0
\end{aligned}
$$

for $i=1,2, \ldots, n$ and $\alpha_{1}, \alpha_{2}, \alpha_{3},=1,2, \ldots, n_{x}$. Here, we apply the tensor notation as in Schmitt-

Grohé \& Uribe (2004). The expression for $\left[b^{1}\right]_{\alpha_{1} \alpha_{2} \alpha_{3}}^{i}$ is stated in the appendix and depends on $\mathbf{g}_{\mathbf{x}}, \mathbf{h}_{\mathbf{x}}, \mathbf{g}_{\mathbf{x} \mathbf{x}}, \mathbf{h}_{\mathbf{x} \mathbf{x}}$ along with first, second, and third order derivatives of $\mathbf{f}$. It is important to note that $[b]_{\alpha_{1} \alpha_{2} \alpha_{3}}^{i}$ is known and different from zero. Hence, the linear system in (7) with $\left(n_{y}+n_{x}\right) \times n_{x} \times n_{x} \times n_{x}$ equations in as many unknowns is straightforward to solve and implies that $\mathbf{g}_{\mathbf{x} \mathbf{x} \mathbf{x}}$ and $\mathbf{h}_{\mathbf{x x \mathbf { x }}}$ are non-zero matrices.

The terms $\mathbf{g}_{\boldsymbol{\sigma} \boldsymbol{\sigma} \mathbf{x}}$ and $\mathbf{h}_{\sigma \sigma \mathbf{x}}$ can be found by differentiating $\mathbf{F}(\mathbf{x}, \sigma)$ twice with respect to $\sigma$ and once with respect to $\mathbf{x}$. Evaluated at $\left(\mathbf{x}_{s s}, 0\right)$ we get

$$
\begin{aligned}
{\left[\mathbf{F}_{\boldsymbol{\sigma} \boldsymbol{\sigma} \mathbf{x}}\left(\mathbf{x}_{s s}, \sigma\right)\right]_{\alpha_{3}}^{i}=} & {\left[\mathbf{f}_{\mathbf{y}^{\prime}}\right]_{\beta_{1}}^{i}\left[\mathbf{g}_{\mathbf{x}}\right]_{\gamma_{1}}^{\beta_{1}}\left[\mathbf{h}_{\sigma \sigma \mathbf{x}}\right]_{\alpha_{3}}^{\gamma_{1}}+\left[\mathbf{f}_{\mathbf{y}^{\prime}}\right]_{\beta_{1}}^{i}\left[\mathbf{g}_{\boldsymbol{\sigma} \boldsymbol{\sigma} \mathbf{x}}\right]_{\gamma_{3}}^{\beta_{1}}\left[\mathbf{h}_{\mathbf{x}}\right]_{\alpha_{3}}^{\gamma_{3}} } \\
& +\left[\mathbf{f}_{\mathbf{y}}\right]_{\beta_{1}}^{i}\left[\mathbf{g}_{\boldsymbol{\sigma} \boldsymbol{\sigma} \mathbf{x}}\right]_{\alpha_{3}}^{\beta_{1}}+\left[\mathbf{f}_{\mathbf{x}^{\prime}}\right]_{\gamma_{1}}^{i}\left[\mathbf{h}_{\boldsymbol{\sigma} \boldsymbol{x} \mathbf{x}}\right]_{\alpha_{3}}^{\gamma_{1}}+\left[b^{2}\right]_{\alpha_{3}}^{i} \\
= & 0
\end{aligned}
$$


for $i=1,2, \ldots, n$ and $\alpha_{3}=1,2, \ldots, n_{x}$. From the expression of $\left[b^{2}\right]_{\alpha_{3}}^{i}$ in the appendix we see that its value is known and non-zero. The latter follows from the fact that second moments of $\boldsymbol{\epsilon}_{t+1}$ are non-zero which also ensure that $\mathbf{g}_{\sigma \sigma}$ and $\mathbf{h}_{\sigma \sigma}$ are non-zero (see Schmitt-Grohé \& Uribe (2004)). As a result, the linear system in (8) with $\left(n_{y}+n_{x}\right) \times n_{x}$ equations in the same number of unknowns allow us to solve for $\mathbf{g}_{\sigma \sigma \mathbf{x}}$ and $\mathbf{h}_{\sigma \sigma \mathbf{x}}$ which in general are non-zero matrices. Hence, a third order approximation to $\mathbf{g}$ and $\mathbf{h}$ imply a correction for uncertainty in terms which are linear in the state vector $\mathbf{x}$. As we will show in the next section, this is the uncertainty correction which generates time-variation in risk premia.

Proceeding in a similar manner, the values of $\mathbf{g}_{\sigma \mathbf{x x}}$ and $\mathbf{h}_{\boldsymbol{\sigma} \mathbf{x x}}$ are given by

$$
\begin{aligned}
{\left[\mathbf{F}_{\boldsymbol{\sigma} \mathbf{x x}}\left(\mathbf{x}_{s s}, \sigma\right)\right]_{\alpha_{2} \alpha_{3}}^{i}=} & {\left[\mathbf{f}_{\mathbf{y}^{\prime}}\right]_{\beta_{1}}^{i}\left[\mathbf{g}_{\mathbf{x}}\right]_{\gamma_{1}}^{\beta_{1}}\left[\mathbf{h}_{\sigma \mathbf{x x}}\right]_{\alpha_{2} \alpha_{3}}^{\gamma_{1}}+\left[\mathbf{f}_{\mathbf{y}^{\prime}}\right]_{\beta_{1}}^{i}\left[\mathbf{g}_{\sigma \mathbf{x x}}\right]_{\gamma_{2} \gamma_{3}}^{\beta_{1}}\left[\mathbf{h}_{\mathbf{x}}\right]_{\alpha_{3}}^{\gamma_{3}}\left[\mathbf{h}_{\mathbf{x}}\right]_{\alpha_{2}}^{\gamma_{2}} } \\
& +\left[\mathbf{f}_{\mathbf{y}}\right]_{\beta_{1}}^{i}\left[\mathbf{g}_{\boldsymbol{\sigma} \mathbf{x} \mathbf{x}}\right]_{\alpha_{2} \alpha_{3}}^{\beta_{1}}+\left[\mathbf{f}_{\mathbf{x}^{\prime}}\right]_{\gamma_{1}}^{i}\left[\mathbf{h}_{\boldsymbol{\sigma} \mathbf{x} \mathbf{x}}\right]_{\alpha_{2} \alpha_{3}}^{\gamma_{1}} \\
= & 0
\end{aligned}
$$

for $i=1,2, \ldots, n$ and $\alpha_{2}, \alpha_{3}=1,2, \ldots, n_{x}$. This linear system of $\left(n_{y}+n_{x}\right) \times n_{x} \times n_{x}$ equations in as many unknowns is homogenous and it therefore follows that $\mathbf{g}_{\boldsymbol{\sigma} \mathbf{x x}}=\mathbf{0}$ and $\mathbf{h}_{\sigma \mathbf{x x}}=\mathbf{0}$. Hence, a third order approximation to $\mathbf{g}$ and $\mathbf{h}$ does not imply a correction for uncertainty in terms which are quadratic in the state vector $\mathbf{x}$.

Finally, the derivatives $\mathbf{g}_{\boldsymbol{\sigma} \boldsymbol{\sigma} \boldsymbol{\sigma}}$ and $\mathbf{h}_{\sigma \sigma \sigma}$ are determined by

$$
\begin{aligned}
{\left[\mathbf{F}_{\sigma \sigma \sigma}\left(\mathbf{x}_{s s}, \sigma\right)\right]^{i}=} & \left(\left[\mathbf{f}_{\mathbf{y}^{\prime}}\right]_{\beta_{1}}^{i}+\left[\mathbf{f}_{\mathbf{y}}\right]_{\beta_{1}}^{i}\right)\left[\mathbf{g}_{\sigma \sigma \sigma}\right]^{\beta_{1}}+\left(\left[\mathbf{f}_{\mathbf{y}^{\prime}}\right]_{\beta_{1}}^{i}\left[\mathbf{g}_{\mathbf{x}}\right]_{\gamma_{1}}^{\beta_{1}}+\left[\mathbf{f}_{\mathbf{y}}\right]_{\beta_{1}}^{i}\right)\left[\mathbf{h}_{\sigma \sigma \sigma}\right]^{\gamma_{1}} \\
& +\left[b^{3}\right]^{i} \\
= & 0
\end{aligned}
$$

for $i=1,2, \ldots, n$. From the expression of $\left[b^{3}\right]^{i}$ in the appendix, we first note that $\left[b^{3}\right]^{i}=0$ if all innovations have symmetric distributions, i.e. all unconditional third moments are zero. In this case, the linear system in (10) is homogenous and we therefore have $\mathbf{g}_{\sigma \sigma \sigma}=\mathbf{0}$ and $\mathbf{h}_{\sigma \sigma \sigma}=\mathbf{0}$. However, if some innovations have non-symmetric distributions, then $\left[b^{3}\right]^{i}$ may be different from zero and $\mathbf{g}_{\sigma \sigma \sigma}$ and $\mathbf{h}_{\sigma \sigma \sigma}$ may therefore also be different from zero. Innovations with this property are widely used in the literature which uses rare disasters to explain asset pricing puzzles (see Rietz (1988), Barro (2006), Gabaix (2008), Barro (2009), among others). Hence, when we allow for rare disasters in DSGE models, the constant terms in a third approximation may require a further correction for uncertainty than implied by the second order terms $\mathbf{g}_{\sigma \sigma}$ and $\mathbf{h}_{\sigma \sigma}$.

This finding is contrary to the conjecture made in Schmitt-Grohé \& Uribe (2004) about $\mathbf{g}_{\sigma \sigma \sigma}$ and $\mathbf{h}_{\sigma \sigma \sigma}$, as they always anticipate these terms to be zero. Schmitt-Grohé \& Uribe (2004) form their conjecture based on numerical results in Aruoba, Fernandez-Villaverde \& Rubio-Ramirez (2006) for the neoclassical growth model with Gaussian distributed innovations where $\mathbf{g}_{\boldsymbol{\sigma} \boldsymbol{\sigma} \boldsymbol{\sigma}}$ and 
$\mathbf{h}_{\sigma \sigma \sigma}$ are zero. It is well-known that the Gaussian distribution is symmetric and our results are therefore in line with their findings. This example illustrates the usefulness of having closed form expressions for the third order terms as inferring their values in general from numerical exercises may be difficult.

We summarize the key results from this section in the following theorem:

Theorem 1 For the class of models in (1)-(2) approximated around the deterministic steady state, it holds that $\mathbf{g}_{\boldsymbol{\sigma} \mathbf{x} \mathbf{x}}=\mathbf{0}$ and $\mathbf{h}_{\boldsymbol{\sigma} \mathbf{x} \mathbf{x}}=\mathbf{0}$. If all innovations have symmetric distributions, then $\mathbf{g}_{\sigma \sigma \sigma}=\mathbf{0}$ and $\mathbf{h}_{\sigma \sigma \sigma}=\mathbf{0}$.

\subsection{Implementation of the derived formulas}

The derived formulas are implemented in a set of Matlab functions which are publicly available on the author's web page or on request. The structure of these codes are explained in the appendix.

We test our derived formulas and their implementation on three examples. Firstly, our codes reproduce the third order terms for the neoclassical growth model computed in the software program Mathematica by Aruoba et al. (2006). Secondly, we also replicate the third order terms in the model by Fernández-Villaverde et al. (2010) which they compute in Mathematica. ${ }^{1}$ The use of symbolic manipulations and solution algorithms for linear systems in Mathematica minimizes the risk of errors, and we therefore consider the two set of Mathematica codes as very reliable benchmarks.

Thirdly, our codes also reproduce the third order terms in the model by Lucas (1978) which Tsionas (2003) solves in closed form for innovations with arbitrary distributions. The correctness of our codes is verified in the case with Gaussian distributed innovations and innovations of the form $u_{t} \equiv 1-\epsilon_{t}$ where $\epsilon_{t}$ is exponential distributed with a mean value of 1 . The latter specification is interesting because the third moment is -2 and leads to a non-zero value of $\mathbf{g}_{\sigma \sigma \sigma}$.

All three tests leave support for the derived formulas and their implementation.

\section{Theoretical results for risk premia}

We first present the general expression for all risk premia at third order in section 4.1. The following three sections then apply our theoretical results from section 3 to study how innovations with non-symmetric distributions, stochastic volatility, and GARCH processes affect risk premia

\footnotetext{
${ }^{1}$ For simplicity, a version of their model without stochastic volitlity is considered. We are grateful to Juan Rubio-Ramirez for providing the third order approximated solution to their model.
} 
in DSGE models approximated up to third order. Innovations with special fourth or fifth moments are not discussed, because only second and third moments of the innovations affected the approximated solution at third order as shown in the previous section.

\subsection{The expression for risk premia at third order}

We start by considering the general expression for all risk premia in DSGE models approximated up to third order around the deterministic steady state. The value of this risk premia is denoted $P_{t}$ and could be equity risk premia, term premia, or exchange rate risk premia. The absence of uncertainty at the steady state means that all risk premia are zero in the approximation point. This further implies that derivatives solely with respect to the state vector, i.e. $P_{\mathbf{x}}, P_{\mathbf{x x}}$, and $P_{\mathbf{x x x}}$, are also zero as these terms do not capture effects of uncertainty. ${ }^{2}$ On the other hand, derivatives with respect to the perturbation parameter $\sigma$ account for the presence of uncertainty and may therefore be non-zero for risk premia. As a result, all risk premia in a third order approximation around the deterministic steady state have the general form

$$
\begin{aligned}
P_{t}= & P_{\sigma} \sigma+\left[\mathbf{P}_{\sigma \mathbf{x}}\right]_{\alpha_{2}} \sigma\left[\mathbf{x}_{t}\right]^{\alpha_{2}}+\frac{1}{2} P_{\sigma \sigma} \sigma^{2} \\
& +\frac{1}{6} P_{\sigma \sigma \sigma} \sigma^{3}+\frac{3}{6}\left[\mathbf{P}_{\sigma \sigma \mathbf{x}}\right]_{\alpha_{3}} \sigma^{2}\left[\mathbf{x}_{t}\right]^{\alpha_{3}}+\frac{3}{6}\left[\mathbf{P}_{\sigma \mathbf{x x}}\right]_{\alpha_{2} \alpha_{3}} \sigma\left[\mathbf{x}_{t}\right]^{\alpha_{2}}\left[\mathbf{x}_{t}\right]^{\alpha_{3}} .
\end{aligned}
$$

for $\alpha_{2}, \alpha_{3}=1,2, \ldots, n_{x}$.

\subsection{Innovations with non-symmetric distributions}

This section examines how risk premia are affected by innovations with non-symmetric distributions, for instance due to rare disasters as in Barro (2006). The second order approximated solution is independent of third order moments as shown in Schmitt-Grohé \& Uribe (2004). We therefore have trivially that innovations with non-symmetric distribution do not affect risk premia at second order.

For a third order approximation, the results in Schmitt-Grohé \& Uribe (2004) imply $P_{\sigma}=0$ and $\mathbf{P}_{\sigma \mathbf{x}}=\mathbf{0}$ and our Theorem 1 gives $\mathbf{P}_{\sigma \mathbf{x x}}=\mathbf{0}$. Hence, the expression for risk premia is given by

$$
P_{t}=\frac{1}{2} P_{\sigma \sigma} \sigma^{2}+\frac{1}{6} P_{\sigma \sigma \sigma} \sigma^{3}+\frac{3}{6}\left[\mathbf{P}_{\sigma \sigma \mathbf{x}}\right]_{\alpha_{3}} \sigma^{2}\left[\mathbf{x}_{t}\right]^{\alpha_{3}} .
$$

Theorem 1 also implies that $P_{\sigma \sigma \sigma}=0$ when all third moments of the innovations are zero, otherwise $P_{\sigma \sigma \sigma} \neq 0$. Accordingly, the mean value of risk premia is affected by the constant $\frac{1}{6} P_{\sigma \sigma \sigma} \sigma^{3}$ when DSGE models have innovations with non-symmetric distributions. Our formulas in section 3 also show that such distributions do not affect the value of $\mathbf{P}_{\sigma \sigma \mathbf{x}}$ because this

\footnotetext{
${ }^{2}$ This property of a perturbation approximation around the steady state may even be taken to be a requirement when defining risk premia.
} 
term only depends on second moments of the innovations. Hence, for a given variance of $\mathbf{x}_{t}$, innovations with non-symmetric distribution do not affect the variability of risk premia which is determined by the term $\frac{3}{6}\left[\mathbf{P}_{\sigma \sigma \mathbf{x}}\right]_{\alpha_{3}} \sigma^{2}\left[\mathbf{x}_{t}\right]^{\alpha_{3}}$. Accordingly, accounting for rare disasters as in Barro (2006) affects the mean level of risk premia but does not generate additional variability in risk premia when DSGE models are solved up to third order. ${ }^{3}$

We summarize these results in the following proposition:

Proposition 1 In a second order approximation, innovations with non-symmetric distributions do not affect risk premia.

Proposition 2 In a third order approximation, innovations with non-symmetric distributions affect the level of risk premia but they do not, for a given variance of $\mathbf{x}_{t}$, affect the variability of risk premia.

\subsection{Stochastic volatility}

Recent work by Fernández-Villaverde \& Rubio-Ramírez (2007) and Justiniano \& Primiceri (2008) have documented the importance of stochastic volatility in the post-war US economy. This section briefly presents their specification of stochastic volatility before deriving the implication of such processes for risk premia in DSGE models.

Justiniano \& Primiceri (2008) use a specification where an exogenous process $a_{t}$ evolves according to

$$
\ln \left(\frac{a_{t+1}}{a_{s s}}\right)=\rho_{a} \ln \left(\frac{a_{t}}{a_{s s}}\right)+\sigma_{a, t+1} \epsilon_{a, t+1},
$$

and $\epsilon_{a, t} \sim \mathcal{N} \mathcal{I D}(0,1)$. In the present discussion, we let $a_{t}$ denote the level of productivity and $a_{s s}$ is the steady state level of $a_{t}$. The difference from the standard log-normal process in the specification by Justiniano \& Primiceri (2008) is that the conditional volatility $\sigma_{a, t}$ in (13) is time-varying and changes according to

$$
\ln \left(\frac{\sigma_{a, t+1}}{\sigma_{a, s s}}\right)=\rho_{\sigma} \ln \left(\frac{\sigma_{a, t}}{\sigma_{a, s s}}\right)+\epsilon_{\sigma, t+1}
$$

where $\epsilon_{\sigma, t} \sim \mathcal{N} \mathcal{I D}\left(0, \operatorname{Var}\left(\epsilon_{\sigma, t}\right)\right)$. The innovations $\epsilon_{a, t+1}$ and $\epsilon_{\sigma, t+1}$ are assumed to be mutually independent at all leads and lags.

A potential problem with stochastic volatility in our framework in (1)-(2) is that $\epsilon_{a, t+1}$ do not enter linearly in (13) as $\epsilon_{a, t+1}$ is scaled by the state variable $\sigma_{a, t+1}$. However, it straightforward

\footnotetext{
${ }^{3}$ Rare disasters may in a fourth order approximation generate additional variation in risk premia because $P_{\sigma \sigma \sigma \mathbf{x}}$ may be non-zero.
} 
to find an equivalent representation of (13) where $\epsilon_{a, t+1}$ enters linearly and therefore fits into our framework. The representation we consider is given by

$$
\begin{gathered}
\ln \left(\frac{a_{t}}{a_{s s}}\right)=\sigma_{a, t} \ln v_{t} \\
\ln v_{t+1}=\rho_{a} \frac{\sigma_{a, t}}{\sigma_{a, t+1}} \ln v_{t}+\epsilon_{a, t+1}
\end{gathered}
$$

where $v_{t}=1$ in the steady state. To see that (15) and (16) are equivalent to (13), first lead (15) by one period and insert (16). This gives

$$
\ln \left(\frac{a_{t+1}}{a_{s s}}\right)=\sigma_{a, t+1}\left(\rho_{a} \frac{\sigma_{a, t}}{\sigma_{a, t+1}} \ln v_{t}+\epsilon_{a, t+1}\right) .
$$

Form (15), $\ln \left(\frac{a_{t}}{a_{s s}}\right) / \sigma_{a, t}=\ln v_{t}$ and (17) therefore simplifies to (13) as claimed. This alternative representation implies that the non-linearity in (13) is moved from the innovations to the local persistency coefficient in the auxiliary process $\ln v_{t}$, and the processes (15) and (16) therefore fit into our framework. ${ }^{4}$

Returning to the implication for risk premia, the results in Schmitt-Grohé \& Uribe (2004) imply $P_{\sigma}=0$ and $\mathbf{P}_{\sigma \mathbf{x}}=\mathbf{0}$, and we therefore have in a second order approximation that

$$
P_{t}=\frac{1}{2} P_{\sigma \sigma} \sigma^{2}
$$

The value of $P_{\sigma \sigma}$ is a linear combination of the second moments to the structural innovations in DSGE models (see Schmitt-Grohé \& Uribe (2004)). Stochastic volatility adds additional state variables and innovations to the model, and this may therefore affect the value of $P_{\sigma \sigma}$. That is stochastic volatility may affect the level of risk premia. We also note that stochastic volatility does not generate time-variation in risk premia at second order because $\mathbf{P}_{\sigma \mathbf{x}}=\mathbf{0}$. This may at first appear surprising because risk premia is a combination of second moments and one could therefore expect that changing the conditional second moments by stochastic volatility would give time-varying risk premia. The reason that this intuitive explanation does not carry through is because all second moments are constant at our approximation point even with stochastic volatility, and the level correction $P_{\sigma \sigma}$ therefore remains constant. In other words, a second order approximation does not capture this effect for risk premia of time-varying second moments.

For a third order approximation, Theorem 1 in section 3 implies the following expression for

\footnotetext{
${ }^{4}$ It is straightforward to show that the alternative specifications of stochastic volatility considered in Andreasen (2010) can be reexpressed in a similar manner. Note also that transformations of shock processes are widely used when DSGE models are solved numerically. For instance, the well-known log-normal process $a_{t+1}=a_{s s}^{1-\rho_{a}} a_{t}^{\rho_{a}} e^{\sigma_{a} \epsilon_{a, t+1}}$ is in most cases represented through a log-transformation as this makes the approximation easier.
} 
risk premia

$$
P_{t}=\frac{1}{2} P_{\sigma \sigma} \sigma^{2}+\frac{1}{6} P_{\sigma \sigma \sigma} \sigma^{3}+\frac{3}{6}\left[\mathbf{P}_{\sigma \sigma \mathbf{x}}\right]_{\alpha_{3}} \sigma^{2}\left[\mathbf{x}_{t}\right]^{\alpha_{3}},
$$

when some innovations have non-zero third moments, otherwise $P_{\sigma \sigma \sigma}=0$ and the expression reduces accordingly. We note that the term $P_{\sigma \sigma \sigma}$ may change if stochastic volatility induces additional innovations with non-symmetric distributions. As mentioned above, stochastic volatility adds the conditional volatilities to the state vector and this induces more terms in $\left[\mathbf{P}_{\sigma \sigma \mathbf{x}}\right]_{\alpha_{3}} \sigma^{2}\left[\mathbf{x}_{t}\right]^{\alpha_{3}}$. In our case, $\sigma_{a, t}$ is an additional state variable which appears as an extra term in $\left[\mathbf{P}_{\sigma \sigma \mathbf{x}}\right]_{\alpha_{3}} \sigma^{2}\left[\mathbf{x}_{t}\right]^{\alpha_{3}}$. Hence, also the variability of risk premia may be affected by the presence of stochastic volatility in DSGE models when a third order approximation is used.

We summarize the results from this section in the next two propositions:

Proposition 3 In a second order approximation, stochastic volatility may affect the level of risk premia but it does not generate time-variation in risk premia.

Proposition 4 In a third order approximation, stochastic volatility may affect the level of risk premia and it may generate additional time-variation in risk premia.

\subsection{GARCH processes}

Following the work of Bollerslev (1986), GARCH processes have emerged as a popular way to specify conditional heteroscedasticity. This section describes how GARCH processes can be introduced in DSGE models before deriving their implications for risk premia.

We consider the implementation of the widely used $\operatorname{GARCH}(1,1)$ model with Gaussian distributed innovations. ${ }^{5}$ One way to include this specification into DSGE models is to let $a_{t}$ evolve according to

$$
\begin{gathered}
\ln \left(\frac{a_{t+1}}{a_{s s}}\right)=\rho_{a} \ln \left(\frac{a_{t}}{a_{s s}}\right)+\sigma_{a, t+1} \epsilon_{a, t+1} \\
\sigma_{a, t+1}^{2}=\sigma_{a, s s}^{2}\left(1-\rho_{1}\right)+\rho_{1} \sigma_{a, t}^{2}+\rho_{2} \sigma_{a, t}^{2} \epsilon_{a, t}^{2}
\end{gathered}
$$

where $\epsilon_{a, t} \sim \mathcal{N} \mathcal{I D}(0,1) .{ }^{6}$ As in the standard log-normal specification of $a_{t}$ without GARCH, we maintain the log-transformation of $a_{t}$ in (19) to ensure positivity of the technology level. Contrary to stochastic volatility, the conditional volatility in $a_{t+1}$ is in a GARCH process determined based on information in period $t$. The law of motion for $\sigma_{t}^{2}$ in (20) follows the specification adopted in Bollerslev (1986). Here, $\sigma_{a, s s}, \rho_{1}, \rho_{2} \geq 0$ are required to ensure non-negativity of $\sigma_{a, t}^{2}$, and wide-sense stationarity of $\sigma_{a, t}^{2}$ requires $\rho_{1}+\rho_{2}<1$.

\footnotetext{
${ }^{5}$ Extensions to the general $\operatorname{GARCH}(\mathrm{p}, \mathrm{q})$ model with potentially non-Gaussian innovations are straightforward.

${ }^{6}$ The constant term in equation $(20)$ is deliberately scaled by $\left(1-\rho_{1}\right)$ because this ensures that $\sigma_{a, t}=\sigma_{a, s s}$ in the deterministic steady state where $\epsilon_{a, t}^{2}=0$.
} 
Using the same type of argument as in section 4.3, an alternative representation of (19) is given by

$$
\begin{gathered}
\ln \left(\frac{a_{t}}{a_{s s}}\right)=\sigma_{a, t} \ln v_{t} \\
\ln v_{t+1}=\rho_{a} \frac{\sigma_{a, t}}{\sigma_{a, t+1}} \ln v_{t}+\epsilon_{a, t+1}
\end{gathered}
$$

The process for $\sigma_{a, t}^{2}$ can therefore be expressed as

$$
\sigma_{a, t+1}^{2}=\sigma_{a, s s}^{2}\left(1-\rho_{1}\right)+\rho_{1} \sigma_{t}^{2}+\rho_{2} \sigma_{a, t}^{2}\left(\ln v_{t}-\rho_{a} \frac{\sigma_{a, t-1}}{\sigma_{a, t}} \ln v_{t-1}\right)^{2} .
$$

This equivalent representation shows that the GARCH process fits into our framework as the innovation $\epsilon_{a, t+1}$ only enters linearly in $(22)$. We also note that the $\operatorname{GARCH}(1,1)$ process for $a_{t}$ induces $\sigma_{a, t}, \sigma_{a, t-1}$, and $v_{t-1}$ as additional state variables.

Based on this alternative representation of GARCH, we clearly have for risk premia that $P_{\sigma}=0$ and $\mathbf{P}_{\sigma \mathbf{x}}=\mathbf{0}$ due to the results in Schmitt-Grohé \& Uribe (2004). That is, for a second order approximation risk premia is given by

$$
P_{t}=\frac{1}{2} P_{\sigma \sigma} \sigma^{2}
$$

The GARCH process adds additional state variables to the model and GARCH may therefore affect the value of $P_{\sigma \sigma}$ and hence the level of risk premia. More importantly, however, GARCH does not generate time-varying risk premia at second order because $\mathbf{P}_{\sigma \mathbf{x}}=\mathbf{0}$. This may seem counter-intuitive because risk premia is a combination of second moments and GARCH generates time-variation in these moments. However, all second moments are constant at our approximation point even with GARCH, and the level correction $P_{\sigma \sigma}$ therefore remains constant. Hence, a second order approximation does not capture this effect in risk premia of time-varying second moments.

For a third order approximation, Theorem 1 in section 3 implies the following expression for risk premia

$$
P_{t}=\frac{1}{2} P_{\sigma \sigma} \sigma^{2}+\frac{1}{6} P_{\sigma \sigma \sigma} \sigma^{3}+\frac{3}{6}\left[\mathbf{P}_{\sigma \sigma \mathbf{x}}\right]_{\alpha_{3}} \sigma^{2}\left[\mathbf{x}_{t}\right]^{\alpha_{3}}
$$

when some innovations have non-zero third moments, otherwise $P_{\sigma \sigma \sigma}=0$ and the expression reduces accordingly. The additional state variables caused by the presence of GARCH induce more terms in $\left[\mathbf{P}_{\sigma \sigma \mathbf{x}}\right]_{\alpha_{3}}\left[\mathbf{x}_{t}\right]^{\alpha_{3}}$, and GARCH may therefore affect the variability of risk premia.

The results from this section are summarized in the following two propositions:

Proposition 5 In a second order approximation, GARCH may affect the level of risk premia but it does not generate time-variation in risk premia. 
Proposition 6 In a third order approximation, GARCH may affect the level of risk premia and it may generate additional time-variation in risk premia.

\section{Quantitative effects for nominal term premia}

This section examines the quantitative effects of innovations with non-symmetric distributions (i.e. rare disasters), stochastic volatility, and GARCH for the nominal term premia in a standard New Keynesian DSGE model solved to third order. We describe the model in section 5.1 and discuss its calibration in section 5.2. The following three sections examine the effects for the 10-year nominal term premium of introducing rare disasters, stochastic volatility, and GARCH into productivity shocks.

\subsection{A New Keynesian model}

We consider a standard New Keynesian model extended with Epstein-Zin-Weil preferences as introduced by Epstein \& Zin (1989) and Weil (1990). These preferences are included because Rudebusch \& Swanson (2009) show that they help an otherwise standard DSGE model generate a more realistic nominal term premium.

Households: Following Rudebusch \& Swanson (2009), the value function $V_{t}$ for the representative household be given by

$$
V_{t} \equiv\left\{\begin{array}{cc}
u_{t}+\beta\left(E_{t}\left[V_{t+1}^{1-\alpha}\right]\right)^{\frac{1}{1-\alpha}} & \text { for } u_{t} \geq 0 \\
u_{t}-\beta\left(E_{t}\left[\left(-V_{t+1}\right)^{1-\alpha}\right]\right)^{\frac{1}{1-\alpha}} & \text { for } u_{t} \leq 0
\end{array} .\right.
$$

with $\beta \in] 0,1[$ and $\alpha \in \mathbb{R} \backslash\{1\}$. As in Binsbergen, Fernandez-Villaverde, Koijen \& RubioRamirez (2010), the periodic utility function is assumed to be

$$
u\left(c_{t}, n_{t}\right) \equiv \frac{\left(c_{t}^{\nu}\left(1-n_{t}\right)^{1-\nu}\right)^{1-\gamma}}{1-\gamma},
$$

where $\gamma \in \mathbb{R} \backslash\{1\}$ and $\nu \in[0,1]$. Here, $c_{t}$ and $n_{t}$ denotes consumption and labor supply, respectively. The specifications in (26) and (27) imply that the intertemporal elasticity of substitution is given by $1 /(1-\nu(1-\gamma))$. A measure of the relative risk aversion which accounts for the leisure decision is $(\gamma+\alpha(1-\gamma)) \nu$ according to Swanson (2010). Hence, the value of $\alpha$ controls the degree of relative risk aversion whereas $\nu$ and $\gamma$ determine the size of the intertemporal elasticity of substitution. 
The budget constraint for the household is given by

$$
E_{t} M_{t, t+1} x_{t+1}+c_{t}=\frac{x_{t}}{\pi_{t}}+w_{t} n_{t}+d_{t}
$$

where $M_{t, t+1}$ is the nominal stochastic discount factor, $x_{t}$ is state-contingent claims, $\pi_{t}$ is inflation, $w_{t}$ is the real wage, and $d_{t}$ is a lump-sum transfer.

The firms: Final output is produced by a perfectly competitive representative firm which uses a continuum of intermediate goods $y_{t}(i)$ and the production function

$$
y_{t}=\left(\int_{0}^{1}\left(y_{t}(i)\right)^{\frac{\eta-1}{\eta}} d i\right)^{\frac{\eta}{\eta-1}}
$$

with $\eta>1$. This implies

$$
y_{t}(i)=\left(\frac{p_{t}(i)}{p_{t}}\right)^{-\eta} y_{t}
$$

where the aggregate price level is given by $p_{t}=\left[\int_{0}^{1}\left(p_{t}(i)\right)^{1-\eta} d i\right]^{\frac{1}{1-\eta}}$.

All intermediate firms produce a slightly differential good using

$$
y_{t}(i)=z_{t} a_{t} \bar{k}^{\theta} n_{t}(i)^{1-\theta}
$$

where $\bar{k}$ and $n_{t}(i)$ denote physical capital and labor services of the $i$ 'th firm, respectively. The variable $a_{t}$ represents exogenous stationary technology shocks specified below. We use $z_{t}$ to capture a deterministic trend in technology, meaning that $\mu_{z, t} \equiv z_{t} / z_{t-1}$ and $\mu_{z, t}=\mu_{z, s s}$ for all $t$. Intermediate firms maximize the net present value of future profit when setting the optimal level of $n_{t}(i)$ and $p_{t}(i)$. Following Rotemberg (1982), we assume quadratic price adjustment costs controlled by $\xi \geq 0$, and the $i$ 'th firm therefore solves

$$
\max _{n_{t}(i), p_{t}(i)} E_{t} \sum_{j=0}^{\infty} M_{t, t+j}\left[\frac{p_{t+j}(i)}{p_{t+j}} y_{t+j}(i)-w_{t+j} n_{t+j}(i)-\frac{\xi}{2}\left(\frac{p_{t+j}(i)}{p_{t+j-1}(i)} \frac{1}{\pi_{s s}}-1\right)^{2} y_{t+j}\right]
$$

subject to (30) and (31).

The central bank: The behavior of the central bank is given by a standard Taylor-rule

$$
r_{t}=r_{s s}\left(1-\rho_{r}\right)+\rho_{r} r_{t-1}+\phi_{\pi} \ln \left(\frac{\pi_{t}}{\pi_{s s}}\right)+\phi_{y} \ln \left(\frac{y_{t}}{z_{t} y_{s s}}\right)+\epsilon_{R, t}
$$

where $r_{t}$ is the continuously compounded nominal rate and $\epsilon_{R, t+1} \sim \mathcal{N} \mathcal{I D}\left(0, \operatorname{Var}\left(\epsilon_{R, t+1}\right)\right)$. Similar to Justiniano \& Primiceri (2008), the output gap in our specification is measured in terms of output in deviation from the balanced growth path for output.

Aggregation: Simple aggregation implies $y_{t}=z_{t} a_{t} \bar{k}^{\theta} n_{t}^{1-\theta}$ because all intermediate firms 
are identical. The presence of a government sector is specified in a standard way by letting $g_{t} z_{t}$ units of output being used for public consumption in every period. The value of $g_{t}$ is exogenously given by

$$
\ln \left(\frac{g_{t+1}}{g_{s s}}\right)=\rho_{g} \ln \left(\frac{g_{t}}{g_{s s}}\right)+\epsilon_{g, t+1},
$$

where $\epsilon_{g, t+1} \sim \mathcal{N} \mathcal{I D}\left(0, \operatorname{Var}\left(\epsilon_{g, t+1}\right)\right)$. Following Rudebusch \& Swanson (2009) we also assume that $\delta \bar{k} z_{t}$ units of output is used in every period to maintain the fixed capital stock. As a result, the aggregate resource constraint is

$$
y_{t}=c_{t}+g_{t} z_{t}+\delta \bar{k} z_{t}
$$

Technology shock: The model is closed by specifying an exogenous process for technology, $a_{t}$. Our benchmark is the log-normal process where

$$
\ln \left(\frac{a_{t+1}}{a_{s s}}\right)=\rho_{a} \ln \left(\frac{a_{t}}{a_{s s}}\right)+\epsilon_{a, t+1},
$$

and $\epsilon_{a, t+1} \sim \mathcal{N} \mathcal{I D}\left(0, \operatorname{Var}\left(\epsilon_{a, t+1}\right)\right)$. The three alternative specifications of $a_{t}$ we consider in this paper are as follows. The first deals with non-symmetric innovations (i.e. rate disasters) where $a_{t}$ evolves as in (35) but $\epsilon_{a, t+1}$ is given by

$$
\tilde{\epsilon}_{a, t+1}=\left\{\begin{array}{cc}
\mathcal{N}(0,1) & \text { with probability } 1-p \\
\varphi & \text { with probability } p
\end{array}\right.
$$

where

$$
\epsilon_{a, t+1}=\frac{\widetilde{\epsilon}_{a, t+1 t+1}-E\left(\widetilde{\epsilon}_{a, t+1 t+1}\right)}{\sqrt{\operatorname{Var}\left(\widetilde{\epsilon}_{a, t+1 t+1}\right)}} .
$$

Hence, negative values of $\varphi$ induce innovations with negative tails, and vice versa for positive values of $\varphi$. Our second specification considers the case where technology shocks display stochastic volatility as described in section 4.3. The final specification analyzes the case where technology evolves as in section 4.4 and a $\operatorname{GARCH}(1,1)$ process controls the conditional volatility in technology.

\subsection{Calibration and benchmark results}

The model is calibrated to match first and second moments for consumption growth, inflation, the 3-month nominal interest rate, the 10-year nominal interest rate, and the 10-year nominal term premium. ${ }^{7}$ Our calibration is fairly standard and summarized in Table 1 . Some of the

\footnotetext{
${ }^{7}$ We use data from the Federal Reserve Bank of St. Louis. The annualized growth rate in consumption is calculated from real consumption expenditures (PCECC96) and expressed in per capita based on the total
} 
coefficients deserve a few comments. Firstly, we let $\nu=0.35$ and $\gamma=2.5$ which give an intertemporal elasticity of substitution of 0.66. The coefficient related to the Epstein-Zin-Weil preferences $\alpha$ is set to 110 as this implies a mean value of 108 annual basis for the 10 -year nominal term premium. ${ }^{8}$ The empirical moment is in the neighborhood of 106 annual basis points according to Rudebusch \& Swanson (2009). Given these values for $\nu, \gamma$, and $\alpha$, the relative risk aversion is 58,6 when using the measure stated in the previous section.

Secondly, $\beta$ is assigned a relatively high value of 0.9995 to get a sufficiently low mean value for the 3 -month nominal interest rate. Our calibration implies a mean value of $5.82 \%$ in annual terms which is close to the empirical mean of $5.59 \%$. Although the value of $\beta$ may appear relatively high, the effective discount factor in the Euler-consumption equation is $\beta \mu_{z, s s}^{\nu(1-\gamma)-1}=0.9919$ due to the deterministic trend in technology and hence fairly standard.

Thirdly, we set the coefficient for the quadratic price adjustment costs $\xi$ to 260 which for a linearization of the model corresponds to a Calvo coefficient of $0.75 .^{9}$

$<$ Table 1 about here $>$

The first two columns in Table 2 show empirical moments and simulated moments for our benchmark model, respectively. We see that the model is fairly successful in matching the standard deviations for consumption growth, inflation, and the two interest rates. The model is also able to produce a sizeable slope for the nominal term structure of 97 basis points, although this value is somewhat lower than the empirical value of 140 basis points. The model struggles when it comes to explaining the variation in the 10-year term premium std $\left(P_{t, 40}\right)$ which is only 2 basis points in the model compared to 54 basis points in the data. The benchmark model in Rudebusch \& Swanson (2009) displays a similar shortcoming.

$<$ Table 2 about here $>$

\subsection{Rare disasters in technology shock}

We use the work by Barro (2006) on rare disasters to calibrate the shape of the non-symmetric distribution in (36). Barro (2006) estimates disasters to happen with a probability of $1.7 \%$ every

population in the US. The annual inflation rate is for consumer prices. The 3-month nominal interest rate is measured by the rate in the secondary market (TB3MS), and the 10-year nominal rate is taken from Gürkaynak, Sack \& Wright (2007). As in Rudebusch \& Swanson (2009), observations for the 10-year interest rate from 1961Q2 to 1971Q3 are calculated by extrapolation of the estimated curves in Gürkaynak et al. (2007). Finally, the first and second moments for the 10-year nominal term premium are from Rudebusch \& Swanson (2009).

${ }^{8}$ The 10-year nominal term premium in our model is computed by the difference between the 10-year interest rate and the yield to maturity on the corresponding risk neutral bond where payments are discounted by the risk free rate instead of the stochastic discount factor.

${ }^{9}$ It is straightforward to show for a linearized model that the general relationship between the Rotemberg parameter $\xi$ and a Calvo parameter $\alpha_{p}$, giving an average duration of prices of $\frac{1}{1-\alpha_{p}}$ periods, is $\xi=$ $\frac{(1-\theta+\eta \theta)(\eta-1) \alpha_{p}}{\left(1-\alpha_{p}\right)(1-\theta)\left(1-\alpha_{p} \beta \mu_{Z, s s}^{\nu(1-\gamma)}\right)}$. Note that the presence of a deterministic trend and decreasing returns to scale in the production function (see Galí (2008)) modify the relation between $\xi$ and $\alpha_{p}$ as stated in Keen \& Wang (2007). 
year which in our quarterly model corresponds to $p=0.0043$. The value of $\varphi$ is initially set as in Barro (2006) to generate a reduction of 0.29 in technology, which in Barro's model gives an equivalent reduction in output.

The third column in Table 2 shows that this calibration of rare disasters hardly changes the standard deviations for consumption growth, inflation, and the two interest rates. A notable effect appears for the mean slope of the term structure which increases from 97 basis points in the benchmark model to 172 basis points. This increase is generated by lowering the mean level of the 3-month rate (from $5.82 \%$ to $5.30 \%$ ) and by increasing the mean level of the 10-year rate (from $6.79 \%$ to $7.02 \%$ ). The economic intuition behind these results are as follows. A positive probability of a large reduction in consumption during a disaster leads the household to increase its level of precautionary saving. The household therefore requires a lower compensation for postponing consumption, and this explains the lower mean level for the 3-month interest rate. Investing in the 10-year nominal bond becomes even more risky with the presence of disasters because such an event generates high inflation which erodes the real value of this bond when its payoff is most needed to maintain a smooth consumption profile. This lowers the equilibrium price level for the 10-year bond which is equivalent to a higher interest rate.

The presence of rare disasters is further seen to increase the 10-year term premium from 1.08 to 1.81 basis points. The variation in this premium is, however, not affected in a third order approximation to our model. As for the higher order moments, we see that rare disasters increase skewness and kurtosis for consumption growth, inflation, and the two interest rates far beyond the values implied by a Gaussian distribution.

Another calibration of rare disasters is to account for the fact that a disaster typically lasts for several periods and not just for one period as initially assumed. In our second calibration, we therefore assume that a disaster lasts for 5 years. Accounting for the trend in output of 1.005 per quarter, a disaster then reduces output by roughly $40 \%$ when compared to the trend level of output over a 5 year period. Column 5 in Table 2 shows that the effects of rare disasters in this calibration are less pronounced. The value of all standard deviations are hardly affected compared to the benchmark model, and the slope and term premium for the 10-year rate only increase by 7 and 6 basis points, respectively. This second calibration broadly matches skewness and kurtosis for consumption growth whereas these moments for inflation and the two interest rates are too low compared to their empirical values.

Overall, we conclude that the presence of rare disasters in DSGE model can have substantial effects on the level of the term premium along with skewness and kurtosis for consumption growth, inflation, and interest rates. However, rare disasters hardly affect the standard deviations of these macro variables. 


\subsection{Stochastic volatility in technology shock}

We use the estimation results in Justiniano \& Primiceri (2008) to calibrate the process for the stochastic volatility in technology. They find $\operatorname{Var}\left(\epsilon_{\sigma, t}\right)$ to be around $0.01^{2}$ and assume $\rho_{a}=1$. We initially choose the same value for $\operatorname{Var}\left(\epsilon_{\sigma, t}\right)$ but let $\rho_{a}=0.99$ to get a highly persistent but stationary process for $\sigma_{a, t}$.

Comparing the second and third columns in Table 3, we see that many of the moments are not affected by the presence of stochastic volatility. The only notable difference is for the standard deviation in the 10-year term premium which increases from 2 basis points in the benchmark model to 14 basis points. The mean value of this premium is, however, not affected by stochastic volatility.

To further explore the effects of stochastic volatility, we next increase the conditional variance of $\sigma_{a, t}$ to $0.03^{2}$. This leads to a slight increase in the variance of consumption growth, inflation, and the two interest rates when compared to the benchmark model. The mean level for the 10year term premium and the slope of the 10-year term structure are still unchanged by stochastic volatility. A key difference, however, is a sizeable increase in the standard deviation of the 10-year term premium from 2 basis points in the benchmark model to 40 basis points. The corresponding sample moment in the data is around 56 basis points, so stochastic volatility clearly brings the model closer to the data along this dimension. Hence, the inability of the benchmark model to generate sufficient variability in the term premium may be due to the omission of time-varying uncertainty in productivity shocks. We also note from Table 3 that this explanation is consistent with the fourth moments in consumption growth, inflation, and the two interest rates, because stochastic volatility increases kurtosis for these variables and also brings these moments closer to the data.

The final column in Table 3 considers the benchmark model with the same unconditional variance in technology as in the model with the second calibration of stochastic volatility. We observe that the increase in the variability of the 10-year term premium and the higher values of kurtosis are not present in this version of the benchmark model. This means that these properties of the model are generated by stochastic volatility and not by the slightly higher variance in technology as induced by our calibration of stochastic volatility.

$<$ Table 3 about here $>$

To explain the reactions in the economy when a volatility shock in technology hits the economy, it is useful to apply the terminology from finance where risk premia are decomposed into a 'market price of risk' and a 'quantity of risk'. Following Cochrane (2001), we define the market price of risk $M P R_{t}$ in period $t$ by $M P R_{t}=\operatorname{Var}_{t}\left(M_{t, t+1}\right) / E_{t}\left(M_{t, t+1}\right)$. This means that 
the term premium $P_{t}$ mechanically can be decomposed as

$$
P_{t}=M P R_{t} \times \frac{P_{t}}{M P R_{t}}
$$

where $\frac{P_{t}}{M P R_{t}}$ then is the quantity of risk. Table 4 shows mean values and standard deviations for $\operatorname{Var}_{t}\left(M_{t, t 1}\right)$, the market price of risk, and the quantity of risk. We see that stochastic volatility does not change the mean level of the market price of risk, whereas the mean level for the quantity of risk increases slightly (from 0.157 to 0.165 ). Larger effects appear for the standard deviations in the market price of risk, which increases from 0.0009 in the benchmark model to 0.0072 with the second calibration of stochastic volatility. That corresponds to a 8 fold increase. The largest and most notable effect, however, is the increase in the standard deviation for the quantity of risk which increases from 0.0049 in the benchmark model to 0.3302 with our second calibration of stochastic volatility. This constitutes a 67 fold increase. Hence, the large standard deviation for the term premium in our model with stochastic volatility is primarily driven by a more volatile quantity of risk. This finding is similar to the implications from classical finance models such as Cox et al. (1985) and Dai \& Singleton (2002), where an increase in the uncertainty level increases the term premium through a higher quantity of risk.

$<$ Table 3 about here $>$

Impulse responses following a volatility shock in technology are reported in Figure 1. We first note that an increase in the uncertainty about the productivity level lowers consumption. This effect arises from the precautionary saving channel as the risk averse household builds up a larger buffer stock during periods of higher uncertainty. As a result, the household requires a lower compensation for postponing consumption, and we therefore observe a fall in the 3 month interest rate. The higher uncertainty level also generates an increase in the 10-year term premium, mainly due to an increase in the quantity of risk, and we therefore see a rise in the 10-year interest rate. Hence, a positive volatility shock in technology increases the slope of the term structure as the household requires a lower compensation for holding bonds with short maturities and a higher compensation for holding bonds with long maturities. Another interesting observation is that consumption falls and the term premium rises after a volatility shock in technology. This shock therefore helps the model generate counter-cyclical variation in the term premium as typically observed in the data.

$<$ Figure 1 about here $>$

To summarize, we find that stochastic volatility in technology shocks can generate sizeable variation in the 10-year term premium without distorting the ability of the model to match a key number of macro economic moments. However, the considered specification of stochastic volatility does not affect the mean level of this premium. 


\subsection{GARCH in technology shock}

The $\operatorname{GARCH}(1,1)$ process in technology is calibrated to mimic the specification of stochastic volatility for $\sigma_{a, t}$ in the previous section. To get a high degree of persistency in $\sigma_{a, t}$, we let the GARCH-coefficient $\rho_{1}$ be 0.95 . Two values are considered for the ARCH-coefficient, $\rho_{2}=0.01$ and $\rho_{2}=0.04$.

Table 4 shows the effects of adding GARCH to our benchmark model. In our first calibration with $\rho_{2}=0.01$ (column 3) we observe an increase in the standard deviations for consumption growth, inflation, and the two interest rates when compared to the benchmark model. The mean slope of the term structure increases from 97 to 115 basis points, and we see a similar increase in the mean of the 10-year term premium (from 108 to 122 basis points). The standard deviation in this premium is still quite low (4 basis points), and all values of skewness and kurtosis are hardly affected given this calibration of GARCH.

In our second calibration where $\rho_{2}=0.04$, the standard deviations of consumption growth, inflation, and the two interest rates increases further as shown in column 4 of Table 4 . The mean slope of the 10-year term structure almost doubles from 97 in the benchmark model to 216 basis points. A similar increase is observed in the 10-year term premium which is 199 basis points in this calibration. We also see a sizeable impact on the standard deviation of the term premium which increases to 22 basis points. The corresponding empirical moment is around 54 basis points, so GARCH brings the benchmark model closer to the data along this dimension.

When interpreting these results it is important to note that GARCH increases the standard deviation of technology from 0.0378 in the benchmark model to 0.0437 in the second calibration. It is therefore interesting to see how much of the impact from GARCH is due to the larger variability in technology. Column 5 in Table 4 addresses this question by displaying moments for our model without GARCH but with a higher variance in technology. We see that the greater variability in technology can explain some of the increase in the standard deviation of consumption growth and all of the additional variation in inflation and the two interest rates. However, the higher variability in technology only explains about $25 \%$ of the increase in the mean slope of the 10-year term structure and about $40 \%$ of the increase in the mean level of the 10-year term premium. More importantly, the variability in the term premium is hardly affected by the larger variance of technology, and this means that basically all of the additional variation in the term premium is due to GARCH. We therefore conclude that the considered specification of GARCH has an independent impact on the level and the variability of the term premium in our model.

$<$ Table 4 about here $>$

Our results indicate that the effect of GARCH differ from those of stochastic volatility. Most importantly, GARCH affects the mean level of the premium (contrary to stochastic volatility), 
and GARCH has in general a smaller impact on the variability of the 10-year term premium when compared to stochastic volatility. The decomposition of the term premium in Table 3 illustrates another difference between the two methods to model conditional volatility in technology shocks. We see that GARCH increases the mean level for the market price of risk (from 0.017 to 0.043 ) and lowers the mean level for the quantity of risk (from 0.157 to 0.117 ) when compared to the benchmark model. Recall that stochastic volatility does not change the level for the market price of risk and marginally raises the mean quantity of risk. Secondly, GARCH increases the variability of the market price of risk and lowers the variation in the quantity of risk. The latter finding is different from the case with stochastic volatility.

The explanation for the different effects of stochastic volatility and GARCH is a follows. Consider the version of our model with GARCH and a sizeable negative shock to the technology level. This causes a large reduction in consumption and an increase in the conditional volatility of technology in the first period after the shock. The high value of the GARCH-coefficient and the absence of negative innovations to $\sigma_{a, t}$ imply that the conditional volatility in technology is high for many periods after the shock. The latter constitutes a risk to the household because the higher volatility increases the probability of another big negative technology shock and hence a further reduction in consumption. We see broadly the opposite effects following a positive technology shock, although the conditional volatility also increases in this case. However, the risk of these two events does to balance out because the risk adverse household is mostly concerned with the first event. As a result, the household requires a higher compensation for entering into financial investments than without GARCH. The same mechanism is not present with stochastic volatility because the volatility level is not automatically high when consumption is low. This feature explains why the market price of risk is higher and more volatile with GARCH than with stochastic volatility.

Impulse response functions for a positive technology shock with GARCH are shown in Figure 2. On impact the conditional volatility in technology is unchanged, and we therefore have the well-known effects of a positive technology shock, i.e. lower nominal interest rates and an increase in consumption. Note also the small decrease in the term premium on impact. In the next period, the conditional volatility increases and this raises the term premium. Hence, we see a positive relationship between consumption and the term premium. Following a negative technology shock, which generates a reduction in consumption, we observe a similar increase in the conditional volatility and an increase in the term premium (not shown). Accordingly, GARCH does not generate additional counter-cyclical variation in the term premium because both negative and positive shocks to technology increase the conditional volatility.

$<$ Figure 2 about here $>$

To summarize, the presence of GARCH in technology shocks increases the mean level and the standard deviation of the 10-year term premium. For a given variance in technology shocks, 
we also find that GARCH affects the variability of consumption growth but not the variability of inflation and interest rates.

\section{Conclusion}

This paper analyzes the effects of non-Gaussian shocks for risk premia in DSGE models approximated to second and third order. Based on an extension of the results in Schmitt-Grohé \& Uribe (2004) to third order, we derive propositions for how rare disasters, stochastic volatility, and GARCH affect any risk premia in a wide class of DSGE models. Our key findings are as

follows. Firstly, the presence of rare disasters does not affect risk premia when DSGE models are solved up to second order, and rare disasters only change the level of risk premia at third order. Secondly, modelling time-varying uncertainty by stochastic volatility and GARCH do not generate variation in risk premia when the model is solved up to second order. Thirdly, for DSGE models approximated up to third order, stochastic volatility and GARCH may affect the level and the variability of risk premia.

The paper also examines the quantitative effects of non-Gaussian shocks in a standard New Keynesian DSGE model where productivity features rare disasters, stochastic volatility, and GARCH. Here, focus is devoted to the 10-year nominal term premium and a third order approximation to the model. We find that the considered specification of rare disasters can have substantial effects on the level of the term premium and values of skewness and kurtosis for several macro variables. However, rare disasters hardly affect the standard deviation of these macro variables. We also find that stochastic volatility can generate sizeable variation in the term premium without distorting the model's ability to match a key number of macro economic moments. The presence of GARCH in technology shocks is found to increase the mean level and the standard deviation of the term premium. For a given variance in technology shocks, we also find that GARCH affects the variance of consumption growth but not the variance of inflation and interest rates.

Although this paper settles with studying the quantitative effects of non-Gaussian shocks in technology, it would also be interesting to see how rare disasters, stochastic volatility, and GARCH in other structural shocks affect the nominal term premium and other risk premia. Another possibility would be to explore the quantitative effects of non-Gaussian shocks in various extensions of the standard New Keynesian model. We leave these and other questions for future research. 


\section{A The third order approximation}

The third order approximation to $\mathbf{g}$ and $\mathbf{h}$ at the deterministic steady state is given by

$$
\begin{aligned}
{[\mathbf{g}(\mathbf{x}, \sigma)]^{\beta_{1}=} } & \mathbf{g}\left(\mathbf{x}_{s s}, 0\right)+\left[\mathbf{g}_{\mathbf{x}}\left(\mathbf{x}_{s s}, 0\right)\right]_{\alpha_{1}}^{\beta_{1}}\left[\left(\mathbf{x}-\mathbf{x}_{s s}\right)\right]^{\alpha_{1}} \\
& +\frac{1}{2}\left[\mathbf{g}_{\mathbf{x x}}\left(\mathbf{x}_{s s}, 0\right)\right]_{\alpha_{1} \alpha_{2}}^{\beta_{1}}\left[\left(\mathbf{x}-\mathbf{x}_{s s}\right)\right]^{\alpha_{1}}\left[\left(\mathbf{x}-\mathbf{x}_{s s}\right)\right]^{\alpha_{2}} \\
& +\frac{1}{2}\left[\mathbf{g}_{\sigma \sigma}\left(\mathbf{x}_{s s}, 0\right)\right]^{\beta_{1}}[\sigma][\sigma] \\
& +\frac{1}{6}\left[\mathbf{g}_{\mathbf{x x x}}\left(\mathbf{x}_{s s}, 0\right)\right]_{\alpha_{1} \alpha_{2} \alpha_{3}}^{\beta_{1}}\left[\left(\mathbf{x}-\mathbf{x}_{s s}\right)\right]^{\alpha_{1}}\left[\left(\mathbf{x}-\mathbf{x}_{s s}\right)\right]^{\alpha_{2}}\left[\left(\mathbf{x}-\mathbf{x}_{s s}\right)\right]^{\alpha_{3}} \\
& +\frac{3}{6}\left[\mathbf{g}_{\sigma \sigma \mathbf{x}}\left(\mathbf{x}_{s s}, 0\right)\right]_{\alpha_{3}}^{\beta_{1}}[\sigma][\sigma]\left[\left(\mathbf{x}-\mathbf{x}_{s s}\right)\right]^{\alpha_{3}} \\
& +\frac{3}{6}\left[\mathbf{g}_{\sigma \mathbf{x x}}\left(\mathbf{x}_{s s}, 0\right)\right]_{\alpha_{2} \alpha_{3}}^{\beta_{1}}[\sigma]\left[\left(\mathbf{x}-\mathbf{x}_{s s}\right)\right]^{\alpha_{2}}\left[\left(\mathbf{x}-\mathbf{x}_{s s}\right)\right]^{\alpha_{3}} \\
& +\frac{1}{6}\left[\mathbf{g}_{\sigma \sigma \sigma}\left(\mathbf{x}_{s s}, 0\right)\right]^{\beta_{1}}[\sigma][\sigma][\sigma]
\end{aligned}
$$

and

$$
\begin{aligned}
{[\mathbf{h}(\mathbf{x}, \sigma)]^{\gamma_{1}=} } & \mathbf{h}\left(\mathbf{x}_{s s}, 0\right)+\left[\mathbf{h}_{\mathbf{x}}\left(\mathbf{x}_{s s}, 0\right)\right]_{\alpha_{1}}^{\gamma_{1}}\left[\left(\mathbf{x}-\mathbf{x}_{s s}\right)\right]^{\alpha_{1}} \\
& +\frac{1}{2}\left[\mathbf{h}_{\mathbf{x x}}\left(\mathbf{x}_{s s}, 0\right)\right]_{\alpha_{1} \alpha_{2}}^{\gamma_{1}}\left[\left(\mathbf{x}-\mathbf{x}_{s s}\right)\right]^{\alpha_{1}}\left[\left(\mathbf{x}-\mathbf{x}_{s s}\right)\right]^{\alpha_{2}} \\
& +\frac{1}{2}\left[\mathbf{h}_{\sigma \sigma}\left(\mathbf{x}_{s s}, 0\right)\right]^{\gamma_{1}}[\sigma][\sigma] \\
& +\frac{1}{6}\left[\mathbf{h}_{\mathbf{x x x}}\left(\mathbf{x}_{s s}, 0\right)\right]_{\alpha_{1} \alpha_{2} \alpha_{3}}^{\gamma_{1}}\left[\left(\mathbf{x}-\mathbf{x}_{s s}\right)\right]^{\alpha_{1}}\left[\left(\mathbf{x}-\mathbf{x}_{s s}\right)\right]^{\alpha_{2}}\left[\left(\mathbf{x}-\mathbf{x}_{s s}\right)\right]^{\alpha_{3}} \\
& +\frac{3}{6}\left[\mathbf{h}_{\sigma \sigma \mathbf{x}}\left(\mathbf{x}_{s s}, 0\right)\right]_{\alpha_{3}}^{\gamma_{1}}[\sigma][\sigma]\left[\left(\mathbf{x}-\mathbf{x}_{s s}\right)\right]^{\alpha_{3}} \\
& +\frac{3}{6}\left[\mathbf{h}_{\sigma \mathbf{x x}}\left(\mathbf{x}_{s s}, 0\right)\right]_{\alpha_{2} \alpha_{3}}^{\gamma_{1}}[\sigma]\left[\left(\mathbf{x}-\mathbf{x}_{s s}\right)\right]^{\alpha_{2}}\left[\left(\mathbf{x}-\mathbf{x}_{s s}\right)\right]^{\alpha_{3}} \\
& +\frac{1}{6}\left[\mathbf{h}_{\sigma \sigma \sigma}\left(\mathbf{x}_{s s}, 0\right)\right]^{\gamma_{1}}[\sigma][\sigma][\sigma]
\end{aligned}
$$

for $\beta_{1}=1,2, \ldots, n_{y}, \gamma_{1}=1,2, \ldots, n_{x}$, and $\alpha_{1}, \alpha_{2}, \alpha_{3}=1,2, \ldots, n_{x}$. Following Schmitt-Grohé \& Uribe (2004), we apply the tensor notation to simplify the summations. The expressions in (38) and (39) have been simplified in two ways. Firstly, Young's theorem implies that the order of differentiation with respect to $\mathbf{x}$ and $\sigma$ is irrelevant when partial derivatives of $\mathbf{g}$ and $\mathbf{h}$ are continuous. Secondly, only non-zero first and second order derivatives of $\mathbf{g}$ and $\mathbf{h}$ are included in (38) and (39).

\section{B Constants for the third order terms}

\section{B.1 The expression for $\left[b^{1}\right]_{\alpha_{1} \alpha_{2} \alpha_{3}}^{i}$}

$$
\begin{aligned}
{\left[b^{1}\right]_{\alpha_{1} \alpha_{2} \alpha_{3}}^{i} } & \equiv \\
& +\left(\left[\mathbf{f}_{\mathbf{y}^{\prime} \mathbf{y}^{\prime} \mathbf{y}^{\prime}}\right]_{\beta_{1} \beta_{2} \beta_{3}}^{i}\left[\mathbf{g}_{\mathbf{x}}\right]_{\gamma_{3}}^{\beta_{3}}\left[\mathbf{h}_{\mathbf{x}}\right]_{\alpha_{3}}^{\gamma_{3}}+\left[\mathbf{f}_{\mathbf{y}^{\prime} \mathbf{y}^{\prime} \mathbf{y}}\right]_{\beta_{1} \beta_{2} \beta_{3}}^{i}\left[\mathbf{g}_{\mathbf{x}}\right]_{\alpha_{3}}^{\beta_{3}}+\left[\mathbf{f}_{\mathbf{y}^{\prime} \mathbf{y}^{\prime} \mathbf{x}^{\prime}}\right]_{\beta_{1} \beta_{2} \gamma_{3}}^{i}\left[\mathbf{h}_{\mathbf{x}}\right]_{\alpha_{3}}^{\gamma_{3}}+\left[\mathbf{f}_{\mathbf{y}^{\prime} \mathbf{y}^{\prime} \mathbf{x}}\right]_{\beta_{1} \beta_{2} \alpha_{3}}^{i}\right)
\end{aligned}
$$




$$
\begin{aligned}
& \times\left[\mathbf{g}_{\mathbf{x}}\right]_{\gamma_{2}}^{\beta_{2}}\left[\mathbf{h}_{\mathbf{x}}\right]_{\alpha_{2}}^{\gamma_{2}}\left[\mathbf{g}_{\mathbf{x}}\right]_{\gamma_{1}}^{\beta_{1}}\left[\mathbf{h}_{\mathbf{x}}\right]_{\alpha_{1}}^{\gamma_{1}} \\
& +\left[\mathbf{f}_{\mathbf{y}^{\prime} \mathbf{y}^{\prime}}\right]_{\beta_{1} \beta_{2}}^{i}\left(\left[\mathbf{g}_{\mathbf{x x}}\right]_{\gamma_{2} \gamma_{3}}^{\beta_{2}}\left[\mathbf{h}_{\mathbf{x}}\right]_{\alpha_{3}}^{\gamma_{3}}\left[\mathbf{h}_{\mathbf{x}}\right]_{\alpha_{2}}^{\gamma_{2}}\left[\mathbf{g}_{\mathbf{x}}\right]_{\gamma_{1}}^{\beta_{1}}\left[\mathbf{h}_{\mathbf{x}}\right]_{\alpha_{1}}^{\gamma_{1}}+\left[\mathbf{g}_{\mathbf{x}}\right]_{\gamma_{2}}^{\beta_{2}}\left[\mathbf{h}_{\mathbf{x x}}\right]_{\alpha_{2} \alpha_{3}}^{\gamma_{2}}\left[\mathbf{g}_{\mathbf{x}}\right]_{\gamma_{1}}^{\beta_{1}}\left[\mathbf{h}_{\mathbf{x}}\right]_{\alpha_{1}}^{\gamma_{1}}\right. \\
& \left.+\left[\mathbf{g}_{\mathbf{x}}\right]_{\gamma_{2}}^{\beta_{2}}\left[\mathbf{h}_{\mathbf{x}}\right]_{\alpha_{2}}^{\gamma_{2}}\left[\mathbf{g}_{\mathbf{x x}}\right]_{\gamma_{1} \gamma_{3}}^{\beta_{1}}\left[\mathbf{h}_{\mathbf{x}}\right]_{\alpha_{3}}^{\gamma_{3}}\left[\mathbf{h}_{\mathbf{x}}\right]_{\alpha_{1}}^{\gamma_{1}}+\left[\mathbf{g}_{\mathbf{x}}\right]_{\gamma_{2}}^{\beta_{2}}\left[\mathbf{h}_{\mathbf{x}}\right]_{\alpha_{2}}^{\gamma_{2}}\left[\mathbf{g}_{\mathbf{x}}\right]_{\gamma_{1}}^{\beta_{1}}\left[\mathbf{h}_{\mathbf{x x}}\right]_{\alpha_{1} \alpha_{3}}^{\gamma_{1}}\right) \\
& +\left(\left[\mathbf{f}_{\mathbf{y}^{\prime} \mathbf{y} \mathbf{y}^{\prime}}\right]_{\beta_{1} \beta_{2} \beta_{3}}^{i}\left[\mathbf{g}_{\mathbf{x}}\right]_{\gamma_{3}}^{\beta_{3}}\left[\mathbf{h}_{\mathbf{x}}\right]_{\alpha_{3}}^{\gamma_{3}}+\left[\mathbf{f}_{\mathbf{y}^{\prime} \mathbf{y y}}\right]_{\beta_{1} \beta_{2} \beta_{3}}^{i}\left[\mathbf{g}_{\mathbf{x}}\right]_{\alpha_{3}}^{\beta_{3}}+\left[\mathbf{f}_{\mathbf{y}^{\prime} \mathbf{y} \mathbf{x}^{\prime}}\right]_{\beta_{1} \beta_{2} \gamma_{3}}^{i}\left[\mathbf{h}_{\mathbf{x}}\right]_{\alpha_{3}}^{\gamma_{3}}+\left[\mathbf{f}_{\mathbf{y}^{\prime} \mathbf{y} \mathbf{x}}\right]_{\beta_{1} \beta_{2} \alpha_{3}}^{i}\right) \\
& \times\left[\mathbf{g}_{\mathbf{x}}\right]_{\alpha_{2}}^{\beta_{2}}\left[\mathbf{g}_{\mathbf{x}}\right]_{\gamma_{1}}^{\beta_{1}}\left[\mathbf{h}_{\mathbf{x}}\right]_{\alpha_{1}}^{\gamma_{1}} \\
& +\left[\mathbf{f}_{\mathbf{y}^{\prime} \mathbf{y}}\right]_{\beta_{1} \beta_{2}}^{i}\left(\left[\mathbf{g}_{\mathbf{x x}}\right]_{\alpha_{2} \alpha_{3}}^{\beta_{2}}\left[\mathbf{g}_{\mathbf{x}}\right]_{\gamma_{1}}^{\beta_{1}}\left[\mathbf{h}_{\mathbf{x}}\right]_{\alpha_{1}}^{\gamma_{1}}+\left[\mathbf{g}_{\mathbf{x}}\right]_{\alpha_{2}}^{\beta_{2}}\left[\mathbf{g}_{\mathbf{x x}}\right]_{\gamma_{1} \gamma_{3}}^{\beta_{1}}\left[\mathbf{h}_{\mathbf{x}}\right]_{\alpha_{3}}^{\gamma_{3}}\left[\mathbf{h}_{\mathbf{x}}\right]_{\alpha_{1}}^{\gamma_{1}}+\left[\mathbf{g}_{\mathbf{x}}\right]_{\alpha_{2}}^{\beta_{2}}\left[\mathbf{g}_{\mathbf{x}}\right]_{\gamma_{1}}^{\beta_{1}}\left[\mathbf{h}_{\mathbf{x x}}\right]_{\alpha_{1} \alpha_{3}}^{\gamma_{1}}\right) \\
& +\left(\left[\mathbf{f}_{\mathbf{y}^{\prime} \mathbf{x}^{\prime} \mathbf{y}^{\prime}}\right]_{\beta_{1} \gamma_{2} \beta_{3}}^{i}\left[\mathbf{g}_{\mathbf{x}}\right]_{\gamma_{3}}^{\beta_{3}}\left[\mathbf{h}_{\mathbf{x}}\right]_{\alpha_{3}}^{\gamma_{3}}+\left[\mathbf{f}_{\mathbf{y}^{\prime} \mathbf{x}^{\prime} \mathbf{y}}\right]_{\beta_{1} \gamma_{2} \beta_{3}}^{i}\left[\mathbf{g}_{\mathbf{x}}\right]_{\alpha_{3}}^{\beta_{3}}+\left[\mathbf{f}_{\mathbf{y}^{\prime} \mathbf{x}^{\prime} \mathbf{x}^{\prime}}\right]_{\beta_{1} \gamma_{2} \gamma_{3}}^{i}\left[\mathbf{h}_{\mathbf{x}}\right]_{\alpha_{3}}^{\gamma_{3}}+\left[\mathbf{f}_{\mathbf{y}^{\prime} \mathbf{x}^{\prime} \mathbf{x}}\right]_{\beta_{1} \gamma_{2} \alpha_{3}}^{i}\right) \\
& \times\left[\mathbf{h}_{\mathbf{x}}\right]_{\alpha_{2}}^{\gamma_{2}}\left[\mathbf{g}_{\mathbf{x}}\right]_{\gamma_{1}}^{\beta_{1}}\left[\mathbf{h}_{\mathbf{x}}\right]_{\alpha_{1}}^{\gamma_{1}} \\
& +\left[\mathbf{f}_{\mathbf{y}^{\prime} \mathbf{x}^{\prime}}\right]_{\beta_{1} \gamma_{2}}^{i}\left(\left[\mathbf{h}_{\mathbf{x x}}\right]_{\alpha_{2} \alpha_{3}}^{\gamma_{2}}\left[\mathbf{g}_{\mathbf{x}}\right]_{\gamma_{1}}^{\beta_{1}}\left[\mathbf{h}_{\mathbf{x}}\right]_{\alpha_{1}}^{\gamma_{1}}+\left[\mathbf{h}_{\mathbf{x}}\right]_{\alpha_{2}}^{\gamma_{2}}\left[\mathbf{g}_{\mathbf{x x}}\right]_{\gamma_{1} \gamma_{3}}^{\beta_{1}}\left[\mathbf{h}_{\mathbf{x}}\right]_{\alpha_{3}}^{\gamma_{3}}\left[\mathbf{h}_{\mathbf{x}}\right]_{\alpha_{1}}^{\gamma_{1}}+\left[\mathbf{h}_{\mathbf{x}}\right]_{\alpha_{2}}^{\gamma_{2}}\left[\mathbf{g}_{\mathbf{x}}\right]_{\gamma_{1}}^{\beta_{1}}\left[\mathbf{h}_{\mathbf{x x}}\right]_{\alpha_{1} \alpha_{3}}^{\gamma_{1}}\right) \\
& +\left(\left[\mathbf{f}_{\mathbf{y}^{\prime} \mathbf{x y} \mathbf{y}^{\prime}}\right]_{\beta_{1} \alpha_{2} \beta_{3}}^{i}\left[\mathbf{g}_{\mathbf{x}}\right]_{\gamma_{3}}^{\beta_{3}}\left[\mathbf{h}_{\mathbf{x}}\right]_{\alpha_{3}}^{\gamma_{3}}+\left[\mathbf{f}_{\mathbf{y}^{\prime} \mathbf{x y}}\right]_{\beta_{1} \alpha_{2} \beta_{3}}^{i}\left[\mathbf{g}_{\mathbf{x}}\right]_{\alpha_{3}}^{\beta_{3}}+\left[\mathbf{f}_{\mathbf{y}^{\prime} \mathbf{x} \mathbf{x}^{\prime}}\right]_{\beta_{1} \alpha_{2} \gamma_{3}}^{i}\left[\mathbf{h}_{\mathbf{x}}\right]_{\alpha_{3}}^{\gamma_{3}}+\left[\mathbf{f}_{\mathbf{y}^{\prime} \mathbf{x x}}\right]_{\beta_{1} \alpha_{2} \alpha_{3}}^{i}\right) \\
& \times\left[\mathbf{g}_{\mathbf{x}}\right]_{\gamma_{1}}^{\beta_{1}}\left[\mathbf{h}_{\mathbf{x}}\right]_{\alpha_{1}}^{\gamma_{1}} \\
& +\left[\mathbf{f}_{\mathbf{y}^{\prime} \mathbf{x}}\right]_{\beta_{1} \alpha_{2}}^{i}\left(\left[\mathbf{g}_{\mathbf{x x}}\right]_{\gamma_{1} \gamma_{3}}^{\beta_{1}}\left[\mathbf{h}_{\mathbf{x}}\right]_{\alpha_{3}}^{\gamma_{3}}\left[\mathbf{h}_{\mathbf{x}}\right]_{\alpha_{1}}^{\gamma_{1}}+\left[\mathbf{g}_{\mathbf{x}}\right]_{\gamma_{1}}^{\beta_{1}}\left[\mathbf{h}_{\mathbf{x x}}\right]_{\alpha_{1} \alpha_{3}}^{\gamma_{1}}\right) \\
& +\left(\left[\mathbf{f}_{\mathbf{y}^{\prime} \mathbf{y}^{\prime}}\right]_{\beta_{1} \beta_{3}}^{i}\left[\mathbf{g}_{\mathbf{x}}\right]_{\gamma_{3}}^{\beta_{3}}\left[\mathbf{h}_{\mathbf{x}}\right]_{\alpha_{3}}^{\gamma_{3}}+\left[\mathbf{f}_{\mathbf{y}^{\prime} \mathbf{y}}\right]_{\beta_{1} \beta_{3}}^{i}\left[\mathbf{g}_{\mathbf{x}}\right]_{\alpha_{3}}^{\beta_{3}}+\left[\mathbf{f}_{\mathbf{y}^{\prime} \mathbf{x}^{\prime}}\right]_{\beta_{1} \gamma_{3}}^{i}\left[\mathbf{h}_{\mathbf{x}}\right]_{\alpha_{3}}^{\gamma_{3}}+\left[\mathbf{f}_{\mathbf{y}^{\prime} \mathbf{x}}\right]_{\beta_{1} \alpha_{3}}^{i}\right) \\
& \times\left[\mathbf{g}_{\mathbf{x x}}\right]_{\gamma_{1} \gamma_{2}}^{\beta_{1}}\left[\mathbf{h}_{\mathbf{x}}\right]_{\alpha_{2}}^{\gamma_{2}}\left[\mathbf{h}_{\mathbf{x}}\right]_{\alpha_{1}}^{\gamma_{1}} \\
& +\left[\mathbf{f}_{\mathbf{y}^{\prime}}\right]_{\beta_{1}}^{i}\left(\left[\mathbf{g}_{\mathbf{x x}}\right]_{\gamma_{1} \gamma_{2}}^{\beta_{1}}\left[\mathbf{h}_{\mathbf{x x}}\right]_{\alpha_{2} \alpha_{3}}^{\gamma_{2}}\left[\mathbf{h}_{\mathbf{x}}\right]_{\alpha_{1}}^{\gamma_{1}}+\left[\mathbf{g}_{\mathbf{x x}}\right]_{\gamma_{1} \gamma_{2}}^{\beta_{1}}\left[\mathbf{h}_{\mathbf{x}}\right]_{\alpha_{2}}^{\gamma_{2}}\left[\mathbf{h}_{\mathbf{x x}}\right]_{\alpha_{1} \alpha_{3}}^{\gamma_{1}}\right) \\
& +\left(\left[\mathbf{f}_{\mathbf{y}^{\prime} \mathbf{y}^{\prime}}\right]_{\beta_{1} \beta_{3}}^{i}\left[\mathbf{g}_{\mathbf{x}}\right]_{\gamma_{3}}^{\beta_{3}}\left[\mathbf{h}_{\mathbf{x}}\right]_{\alpha_{3}}^{\gamma_{3}}+\left[\mathbf{f}_{\mathbf{y}^{\prime} \mathbf{y}}\right]_{\beta_{1} \beta_{3}}^{i}\left[\mathbf{g}_{\mathbf{x}}\right]_{\alpha_{3}}^{\beta_{3}}+\left[\mathbf{f}_{\mathbf{y}^{\prime} \mathbf{x}^{\prime}}\right]_{\beta_{1} \gamma_{3}}^{i}\left[\mathbf{h}_{\mathbf{x}}\right]_{\alpha_{3}}^{\gamma_{3}}+\left[\mathbf{f}_{\mathbf{y}^{\prime} \mathbf{x}}\right]_{\beta_{1} \alpha_{3}}^{i}\right) \\
& \times\left[\mathbf{g}_{\mathbf{x}}\right]_{\gamma_{1}}^{\beta_{1}}\left[\mathbf{h}_{\mathbf{x x}}\right]_{\alpha_{1} \alpha_{2}}^{\gamma_{1}} \\
& +\left[\mathbf{f}_{\mathbf{y}^{\prime}}\right]_{\beta_{1}}^{i}\left[\mathbf{g}_{\mathbf{x x}}\right]_{\gamma_{1} \gamma_{3}}^{\beta_{1}}\left[\mathbf{h}_{\mathbf{x}}\right]_{\alpha_{3}}^{\gamma_{3}}\left[\mathbf{h}_{\mathbf{x x}}\right]_{\alpha_{1} \alpha_{2}}^{\gamma_{1}} \\
& +\left(\left[\mathbf{f}_{\mathbf{y} \mathbf{y}^{\prime} \mathbf{y}^{\prime}}\right]_{\beta_{1} \beta_{2} \beta_{3}}^{i}\left[\mathbf{g}_{\mathbf{x}}\right]_{\gamma_{3}}^{\beta_{3}}\left[\mathbf{h}_{\mathbf{x}}\right]_{\alpha_{3}}^{\gamma_{3}}+\left[\mathbf{f}_{\mathbf{y} \mathbf{y}^{\prime} \mathbf{y}}\right]_{\beta_{1} \beta_{2} \beta_{3}}^{i}\left[\mathbf{g}_{\mathbf{x}}\right]_{\alpha_{3}}^{\beta_{3}}+\left[\mathbf{f}_{\mathbf{y} \mathbf{y}^{\prime} \mathbf{x}^{\prime}}\right]_{\beta_{1} \beta_{2} \gamma_{3}}^{i}\left[\mathbf{h}_{\mathbf{x}}\right]_{\alpha_{3}}^{\gamma_{3}}+\left[\mathbf{f}_{\mathbf{y} \mathbf{y}^{\prime} \mathbf{x}}\right]_{\beta_{1} \beta_{2} \alpha_{3}}^{i}\right) \\
& \times\left[\mathbf{g}_{\mathbf{x}}\right]_{\gamma_{2}}^{\beta_{2}}\left[\mathbf{h}_{\mathbf{x}}\right]_{\alpha_{2}}^{\gamma_{2}}\left[\mathbf{g}_{\mathbf{x}}\right]_{\alpha_{1}}^{\beta_{1}} \\
& +\left[\mathbf{f}_{\mathbf{y} \mathbf{y}^{\prime}}\right]_{\beta_{1} \beta_{2}}^{i}\left(\left[\mathbf{g}_{\mathbf{x x}}\right]_{\gamma_{2} \gamma_{3}}^{\beta_{2}}\left[\mathbf{h}_{\mathbf{x}}\right]_{\alpha_{3}}^{\gamma_{3}}\left[\mathbf{h}_{\mathbf{x}}\right]_{\alpha_{2}}^{\gamma_{2}}\left[\mathbf{g}_{\mathbf{x}}\right]_{\alpha_{1}}^{\beta_{1}}+\left[\mathbf{g}_{\mathbf{x}}\right]_{\gamma_{2}}^{\beta_{2}}\left[\mathbf{h}_{\mathbf{x x}}\right]_{\alpha_{2} \alpha_{3}}^{\gamma_{2}}\left[\mathbf{g}_{\mathbf{x}}\right]_{\alpha_{1}}^{\beta_{1}}+\left[\mathbf{g}_{\mathbf{x}}\right]_{\gamma_{2}}^{\beta_{2}}\left[\mathbf{h}_{\mathbf{x}}\right]_{\alpha_{2}}^{\gamma_{2}}\left[\mathbf{g}_{\mathbf{x x}}\right]_{\alpha_{1} \alpha_{3}}^{\beta_{1}}\right) \\
& +\left(\left[\mathbf{f}_{\mathbf{y y y} \mathbf{y}^{\prime}}\right]_{\beta_{1} \beta_{2} \beta_{3}}^{i}\left[\mathbf{g}_{\mathbf{x}}\right]_{\gamma_{3}}^{\beta_{3}}\left[\mathbf{h}_{\mathbf{x}}\right]_{\alpha_{3}}^{\gamma_{3}}+\left[\mathbf{f}_{\mathbf{y y y}}\right]_{\beta_{1} \beta_{2} \beta_{3}}^{i}\left[\mathbf{g}_{\mathbf{x}}\right]_{\alpha_{3}}^{\beta_{3}}+\left[\mathbf{f}_{\mathbf{y y x} \mathbf{x}^{\prime}}\right]_{\beta_{1} \beta_{2} \gamma_{3}}^{i}\left[\mathbf{h}_{\mathbf{x}}\right]_{\alpha_{3}}^{\gamma_{3}}+\left[\mathbf{f}_{\mathbf{y y x}}\right]_{\beta_{1} \beta_{2} \alpha_{3}}^{i}\right) \\
& \times\left[\mathbf{g}_{\mathbf{x}}\right]_{\alpha_{2}}^{\beta_{2}}\left[\mathbf{g}_{\mathbf{x}}\right]_{\alpha_{1}}^{\beta_{1}} \\
& +\left[\mathbf{f}_{\mathbf{y y}}\right]_{\beta_{1} \beta_{2}}^{i}\left(\left[\mathbf{g}_{\mathbf{x x}}\right]_{\alpha_{2} \alpha_{3}}^{\beta_{2}}\left[\mathbf{g}_{\mathbf{x}}\right]_{\alpha_{1}}^{\beta_{1}}+\left[\mathbf{g}_{\mathbf{x}}\right]_{\alpha_{2}}^{\beta_{2}}\left[\mathbf{g}_{\mathbf{x x}}\right]_{\alpha_{1} \alpha_{3}}^{\beta_{1}}\right) \\
& +\left(\left[\mathbf{f}_{\mathbf{y} \mathbf{x}^{\prime} \mathbf{y}^{\prime}}\right]_{\beta_{1} \gamma_{2} \beta_{3}}^{i}\left[\mathbf{g}_{\mathbf{x}}\right]_{\gamma_{3}}^{\beta_{3}}\left[\mathbf{h}_{\mathbf{x}}^{t}\right]_{\alpha_{3}}^{\gamma_{3}}+\left[\mathbf{f}_{\mathbf{y} \mathbf{x}^{\prime} \mathbf{y}}\right]_{\beta_{1} \gamma_{2} \beta_{3}}^{i}\left[\mathbf{g}_{\mathbf{x}}\right]_{\alpha_{3}}^{\beta_{3}}+\left[\mathbf{f}_{\mathbf{y} \mathbf{x}^{\prime} \mathbf{x}^{\prime}}\right]_{\beta_{1} \gamma_{2} \gamma_{3}}^{i}\left[\mathbf{h}_{\mathbf{x}}\right]_{\alpha_{3}}^{\gamma_{3}}+\left[\mathbf{f}_{\mathbf{y} \mathbf{x}^{\prime} \mathbf{x}}\right]_{\beta_{1} \gamma_{2} \alpha_{3}}^{i}\right) \\
& \times\left[\mathbf{h}_{\mathbf{x}}\right]_{\alpha_{2}}^{\gamma_{2}}\left[\mathbf{g}_{\mathbf{x}}\right]_{\alpha_{1}}^{\beta_{1}} \\
& +\left[\mathbf{f}_{\mathbf{y x} \mathbf{x}^{\prime}}\right]_{\beta_{1} \gamma_{2}}^{i}\left(\left[\mathbf{h}_{\mathbf{x x}}\right]_{\alpha_{2} \alpha_{3}}^{\gamma_{2}}\left[\mathbf{g}_{\mathbf{x}}\right]_{\alpha_{1}}^{\beta_{1}}+\left[\mathbf{h}_{\mathbf{x}}\right]_{\alpha_{2}}^{\gamma_{2}}\left[\mathbf{g}_{\mathbf{x x}}\right]_{\alpha_{1} \alpha_{3}}^{\beta_{1}}\right) \\
& +\left(\left[\mathbf{f}_{\mathbf{y x y}}\right]_{\beta_{1} \alpha_{2} \beta_{3}}^{i}\left[\mathbf{g}_{\mathbf{x}}\right]_{\gamma_{3}}^{\beta_{3}}\left[\mathbf{h}_{\mathbf{x}}\right]_{\alpha_{3}}^{\gamma_{3}}+\left[\mathbf{f}_{\mathbf{y x y}}\right]_{\beta_{1} \alpha_{2} \beta_{3}}^{i}\left[\mathbf{g}_{\mathbf{x}}\right]_{\alpha_{3}}^{\beta_{3}}+\left[\mathbf{f}_{\mathbf{y x x} \mathbf{x}^{\prime}}\right]_{\beta_{1} \alpha_{2} \gamma_{3}}^{i}\left[\mathbf{h}_{\mathbf{x}}\right]_{\alpha_{3}}^{\gamma_{3}}+\left[\mathbf{f}_{\mathbf{y x x}}\right]_{\beta_{1} \alpha_{2} \alpha_{3}}^{i}\right)\left[\mathbf{g}_{\mathbf{x}}\right]_{\alpha_{1}}^{\beta_{1}} \\
& +\left[\mathbf{f}_{\mathbf{y x}}\right]_{\beta_{1} \alpha_{2}}^{i}\left[\mathbf{g}_{\mathbf{x x}}\right]_{\alpha_{1} \alpha_{3}}^{\beta_{1}} \\
& +\left(\left[\mathbf{f}_{\mathbf{y} \mathbf{y}^{\prime}}\right]_{\beta_{1} \beta_{3}}^{i}\left[\mathbf{g}_{\mathbf{x}}\right]_{\gamma_{3}}^{\beta_{3}}\left[\mathbf{h}_{\mathbf{x}}\right]_{\alpha_{3}}^{\gamma_{3}}+\left[\mathbf{f}_{\mathbf{y y}}\right]_{\beta_{1} \beta_{3}}^{i}\left[\mathbf{g}_{\mathbf{x}}\right]_{\alpha_{3}}^{\beta_{3}}+\left[\mathbf{f}_{\mathbf{y} \mathbf{x}^{\prime}}\right]_{\beta_{1} \gamma_{3}}^{i}\left[\mathbf{h}_{\mathbf{x}}\right]_{\alpha_{3}}^{\gamma_{3}}+\left[\mathbf{f}_{\mathbf{y} \mathbf{x}}\right]_{\beta_{1} \alpha_{3}}^{i}\right)\left[\mathbf{g}_{\mathbf{x x}}\right]_{\alpha_{1} \alpha_{2}}^{\beta_{1}} \\
& +\left(\left[\mathbf{f}_{\mathbf{x}^{\prime} \mathbf{y}^{\prime} \mathbf{y}^{\prime}}\right]_{\gamma_{1} \beta_{2} \beta_{3}}^{i}\left[\mathbf{g}_{\mathbf{x}}\right]_{\gamma_{3}}^{\beta_{3}}\left[\mathbf{h}_{\mathbf{x}}\right]_{\alpha_{3}}^{\gamma_{3}}+\left[\mathbf{f}_{\mathbf{x}^{\prime} \mathbf{y}^{\prime} \mathbf{y}}\right]_{\gamma_{1} \beta_{2} \beta_{3}}^{i}\left[\mathbf{g}_{\mathbf{x}}\right]_{\alpha_{3}}^{\beta_{3}}+\left[\mathbf{f}_{\mathbf{x}^{\prime} \mathbf{y}^{\prime} \mathbf{x}^{\prime}}\right]_{\gamma_{1} \beta_{2} \gamma_{3}}^{i}\left[\mathbf{h}_{\mathbf{x}}\right]_{\alpha_{3}}^{\gamma_{3}}+\left[\mathbf{f}_{\mathbf{x}^{\prime} \mathbf{y}^{\prime} \mathbf{x}}\right]_{\gamma_{1} \beta_{2} \alpha_{3}}^{i}\right) \\
& \times\left[\mathbf{g}_{\mathbf{x}}\right]_{\gamma_{2}}^{\beta_{2}}\left[\mathbf{h}_{\mathbf{x}}\right]_{\alpha_{2}}^{\gamma_{2}}\left[\mathbf{h}_{\mathbf{x}}\right]_{\alpha_{1}}^{\gamma_{1}} \\
& +\left[\mathbf{f}_{\mathbf{x}^{\prime} \mathbf{y}^{\prime}}\right]_{\gamma_{1} \beta_{2}}^{i}\left(\left[\mathbf{g}_{\mathbf{x x}}\right]_{\gamma_{2} \gamma_{3}}^{\beta_{2}}\left[\mathbf{h}_{\mathbf{x}}\right]_{\alpha_{3}}^{\gamma_{3}}\left[\mathbf{h}_{\mathbf{x}}\right]_{\alpha_{2}}^{\gamma_{2}}\left[\mathbf{h}_{\mathbf{x}}\right]_{\alpha_{1}}^{\gamma_{1}}+\left[\mathbf{g}_{\mathbf{x}}\right]_{\gamma_{2}}^{\beta_{2}}\left[\mathbf{h}_{\mathbf{x x}}\right]_{\alpha_{2} \alpha_{3}}^{\gamma_{2}}\left[\mathbf{h}_{\mathbf{x}}\right]_{\alpha_{1}}^{\gamma_{1}}+\left[\mathbf{g}_{\mathbf{x}}\right]_{\gamma_{2}}^{\beta_{2}}\left[\mathbf{h}_{\mathbf{x}}\right]_{\alpha_{2}}^{\gamma_{2}}\left[\mathbf{h}_{\mathbf{x x}}\right]_{\alpha_{1} \alpha_{3}}^{\gamma_{1}}\right)
\end{aligned}
$$




$$
\begin{aligned}
& +\left(\left[\mathbf{f}_{\mathbf{x}^{\prime} \mathbf{y} \mathbf{y}^{\prime}}\right]_{\gamma_{1} \beta_{2} \beta_{3}}^{i}\left[\mathbf{g}_{\mathbf{x}}\right]_{\gamma_{3}}^{\beta_{3}}\left[\mathbf{h}_{\mathbf{x}}\right]_{\alpha_{3}}^{\gamma_{3}}+\left[\mathbf{f}_{\mathbf{x}^{\prime} \mathbf{y y}}\right]_{\gamma_{1} \beta_{2} \beta_{3}}^{i}\left[\mathbf{g}_{\mathbf{x}}\right]_{\alpha_{3}}^{\beta_{3}}+\left[\mathbf{f}_{\mathbf{x}^{\prime} \mathbf{y} \mathbf{x}^{\prime}}\right]_{\gamma_{1} \beta_{2} \gamma_{3}}^{i}\left[\mathbf{h}_{\mathbf{x}}\right]_{\alpha_{3}}^{\gamma_{3}}+\left[\mathbf{f}_{\mathbf{x}^{\prime} \mathbf{y x}}\right]_{\gamma_{1} \beta_{2} \alpha_{3}}^{i}\right) \\
& \times\left[\mathbf{g}_{\mathbf{x}}\right]_{\alpha_{2}}^{\beta_{2}}\left[\mathbf{h}_{\mathbf{x}}\right]_{\alpha_{1}}^{\gamma_{1}} \\
& +\left[\mathbf{f}_{\mathbf{x}^{\prime} \mathbf{y}}\right]_{\gamma_{1} \beta_{2}}^{i}\left(\left[\mathbf{g}_{\mathbf{x x}}\right]_{\alpha_{2} \alpha_{3}}^{\beta_{2}}\left[\mathbf{h}_{\mathbf{x}}\right]_{\alpha_{1}}^{\gamma_{1}}+\left[\mathbf{g}_{\mathbf{x}}\right]_{\alpha_{2}}^{\beta_{2}}\left[\mathbf{h}_{\mathbf{x x}}\right]_{\alpha_{1} \alpha_{3}}^{\gamma_{1}}\right) \\
& +\left(\left[\mathbf{f}_{\mathbf{x}^{\prime} \mathbf{x}^{\prime} \mathbf{y}^{\prime}}\right]_{\gamma_{1} \gamma_{2} \beta_{3}}^{i}\left[\mathbf{g}_{\mathbf{x}}\right]_{\gamma_{3}}^{\beta_{3}}\left[\mathbf{h}_{\mathbf{x}}\right]_{\alpha_{3}}^{\gamma_{3}}+\left[\mathbf{f}_{\mathbf{x}^{\prime} \mathbf{x}^{\prime} \mathbf{y}}\right]_{\gamma_{1} \gamma_{2} \beta_{3}}^{i}\left[\mathbf{g}_{\mathbf{x}}\right]_{\alpha_{3}}^{\beta_{3}}+\left[\mathbf{f}_{\mathbf{x}^{\prime} \mathbf{x}^{\prime} \mathbf{x}^{\prime}}\right]_{\gamma_{1} \gamma_{2} \gamma_{3}}^{i}\left[\mathbf{h}_{\mathbf{x}}\right]_{\alpha_{3}}^{\gamma_{3}}+\left[\mathbf{f}_{\mathbf{x}^{\prime} \mathbf{x}^{\prime} \mathbf{x}}\right]_{\gamma_{1} \gamma_{2} \alpha_{3}}^{i}\right) \\
& \times\left[\mathbf{h}_{\mathbf{x}}\right]_{\alpha_{2}}^{\gamma_{2}}\left[\mathbf{h}_{\mathbf{x}}\right]_{\alpha_{1}}^{\gamma_{1}} \\
& +\left[\mathbf{f}_{\mathbf{x}^{\prime} \mathbf{x}^{\prime}}\right]_{\gamma_{1} \gamma_{2}}^{i}\left(\left[\mathbf{h}_{\mathbf{x x}}\right]_{\alpha_{2} \alpha_{3}}^{\gamma_{2}}\left[\mathbf{h}_{\mathbf{x}}\right]_{\alpha_{1}}^{\gamma_{1}}+\left[\mathbf{h}_{\mathbf{x}}\right]_{\alpha_{2}}^{\gamma_{2}}\left[\mathbf{h}_{\mathbf{x x}}\right]_{\alpha_{1} \alpha_{3}}^{\gamma_{1}}\right) \\
& +\left(\left[\mathbf{f}_{\mathbf{x}^{\prime} \mathbf{x} \mathbf{x}^{\prime}}\right]_{\gamma_{1} \alpha_{2} \beta_{3}}^{i}\left[\mathbf{g}_{\mathbf{x}}\right]_{\gamma_{3}}^{\beta_{3}}\left[\mathbf{h}_{\mathbf{x}}\right]_{\alpha_{3}}^{\gamma_{3}}+\left[\mathbf{f}_{\mathbf{x}^{\prime} \mathbf{x y}}\right]_{\gamma_{1} \alpha_{2} \beta_{3}}^{i}\left[\mathbf{g}_{\mathbf{x}}\right]_{\alpha_{3}}^{\beta_{3}}+\left[\mathbf{f}_{\mathbf{x}^{\prime} \mathbf{x} \mathbf{x}^{\prime}}\right]_{\gamma_{1} \alpha_{2} \gamma_{3}}^{i}\left[\mathbf{h}_{\mathbf{x}}\right]_{\alpha_{3}}^{\gamma_{3}}+\left[\mathbf{f}_{\mathbf{x}^{\prime} \mathbf{x} \mathbf{x}}\right]_{\gamma_{1} \alpha_{2} \alpha_{3}}^{i}\right)\left[\mathbf{h}_{\mathbf{x}}\right]_{\alpha_{1}}^{\gamma_{1}} \\
& +\left[\mathbf{f}_{\mathbf{x}^{\prime} \mathbf{x}}\right]_{\gamma_{1} \alpha_{2}}^{i}\left[\mathbf{h}_{\mathbf{x x}}\right]_{\alpha_{1} \alpha_{3}}^{\gamma_{1}} \\
& +\left(\left[\mathbf{f}_{\mathbf{x}^{\prime} \mathbf{y}^{\prime}}\right]_{\gamma_{1} \beta_{3}}^{i}\left[\mathbf{g}_{\mathbf{x}}\right]_{\gamma_{3}}^{\beta_{3}}\left[\mathbf{h}_{\mathbf{x}}\right]_{\alpha_{3}}^{\gamma_{3}}+\left[\mathbf{f}_{\mathbf{x}^{\prime} \mathbf{y}}\right]_{\gamma_{1} \beta_{3}}^{i}\left[\mathbf{g}_{\mathbf{x}}\right]_{\alpha_{3}}^{\beta_{3}}+\left[\mathbf{f}_{\left.\mathbf{x}^{\prime} \mathbf{x}^{\prime}\right]_{\gamma_{1} \gamma_{3}}^{i}}^{i}\left[\mathbf{h}_{\mathbf{x}}\right]_{\alpha_{3}}^{\gamma_{3}}+\left[\mathbf{f}_{\mathbf{x}^{\prime} \mathbf{x}}\right]_{\gamma_{1} \alpha_{3}}^{i}\right)\left[\mathbf{h}_{\mathbf{x x}}\right]_{\alpha_{1} \alpha_{2}}^{\gamma_{1}}\right. \\
& +\left(\left[\mathbf{f}_{\mathbf{x y}^{\prime} \mathbf{y}^{\prime}}\right]_{\alpha_{1} \beta_{2} \beta_{3}}^{i}\left[\mathbf{g}_{\mathbf{x}}\right]_{\gamma_{3}}^{\beta_{3}}\left[\mathbf{h}_{\mathbf{x}}\right]_{\alpha_{3}}^{\gamma_{3}}+\left[\mathbf{f}_{\mathbf{x y} \mathbf{y}^{\prime} \mathbf{y}}\right]_{\alpha_{1} \beta_{2} \beta_{3}}^{i}\left[\mathbf{g}_{\mathbf{x}}\right]_{\alpha_{3}}^{\beta_{3}}+\left[\mathbf{f}_{\mathbf{x y}} \mathbf{x}^{\prime} \mathbf{x}^{\prime}\right]_{\alpha_{1} \beta_{2} \gamma_{3}}^{i}\left[\mathbf{h}_{\mathbf{x}}\right]_{\alpha_{3}}^{\gamma_{3}}+\left[\mathbf{f}_{\mathbf{x y}^{\prime} \mathbf{x}}\right]_{\alpha_{1} \beta_{2} \alpha_{3}}^{i}\right) \\
& \times\left[\mathbf{g}_{\mathbf{x}}\right]_{\gamma_{2}}^{\beta_{2}}\left[\mathbf{h}_{\mathbf{x}}\right]_{\alpha_{2}}^{\gamma_{2}} \\
& +\left[\mathbf{f}_{\mathbf{x y}}\right]_{\alpha_{1} \beta_{2}}^{i}\left(\left[\mathbf{g}_{\mathbf{x x}}\right]_{\gamma_{2} \gamma_{3}}^{\beta_{2}}\left[\mathbf{h}_{\mathbf{x}}\right]_{\alpha_{3}}^{\gamma_{3}}\left[\mathbf{h}_{\mathbf{x}}\right]_{\alpha_{2}}^{\gamma_{2}}+\left[\mathbf{g}_{\mathbf{x}}\right]_{\gamma_{2}}^{\beta_{2}}\left[\mathbf{h}_{\mathbf{x x}}\right]_{\alpha_{2} \alpha_{3}}^{\gamma_{2}}\right) \\
& +\left(\left[\mathbf{f}_{\mathbf{x y y}^{\prime}}\right]_{\alpha_{1} \beta_{2} \beta_{3}}^{i}\left[\mathbf{g}_{\mathbf{x}}\right]_{\gamma_{3}}^{\beta_{3}}\left[\mathbf{h}_{\mathbf{x}}\right]_{\alpha_{3}}^{\gamma_{3}}+\left[\mathbf{f}_{\mathbf{x y y}}\right]_{\alpha_{1} \beta_{2} \beta_{3}}^{i}\left[\mathbf{g}_{\mathbf{x}}\right]_{\alpha_{3}}^{\beta_{3}}+\left[\mathbf{f}_{\mathbf{x y x}_{t+1}}\right]_{\alpha_{1} \beta_{2} \gamma_{3}}^{i}\left[\mathbf{h}_{\mathbf{x}}\right]_{\alpha_{3}}^{\gamma_{3}}+\left[\mathbf{f}_{\mathbf{x y x}}\right]_{\alpha_{1} \beta_{2} \alpha_{3}}^{i}\right)\left[\mathbf{g}_{\mathbf{x}}\right]_{\alpha_{2}}^{\beta_{2}} \\
& +\left[\mathbf{f}_{\mathbf{x y}}\right]_{\alpha_{1} \beta_{2}}^{i}\left[\mathbf{g}_{\mathbf{x x}}\right]_{\alpha_{2} \alpha_{3}}^{\beta_{2}} \\
& +\left(\left[\mathbf{f}_{\mathbf{x x}^{\prime} \mathbf{y}^{\prime}}\right]_{\alpha_{1} \gamma_{2} \beta_{3}}^{i}\left[\mathbf{g}_{\mathbf{x}}\right]_{\gamma_{3}}^{\beta_{3}}\left[\mathbf{h}_{\mathbf{x}}\right]_{\alpha_{3}}^{\gamma_{3}}+\left[\mathbf{f}_{\mathbf{x x} \mathbf{x}^{\prime} \mathbf{y}}\right]_{\alpha_{1} \gamma_{2} \beta_{3}}^{i}\left[\mathbf{g}_{\mathbf{x}}\right]_{\alpha_{3}}^{\beta_{3}}+\left[\mathbf{f}_{\mathbf{x x}^{\prime} \mathbf{x}^{\prime}}\right]_{\alpha_{1} \gamma_{2} \gamma_{3}}^{i}\left[\mathbf{h}_{\mathbf{x}}\right]_{\alpha_{3}}^{\gamma_{3}}+\left[\mathbf{f}_{\mathbf{x x}^{\prime} \mathbf{x}}\right]_{\alpha_{1} \gamma_{2} \alpha_{3}}^{i}\right)\left[\mathbf{h}_{\mathbf{x}}\right]_{\alpha_{2}}^{\gamma_{2}} \\
& +\left[\mathbf{f}_{\mathbf{x x}^{\prime}}\right]_{\alpha_{1} \gamma_{2}}^{i}\left[\mathbf{h}_{\mathbf{x x}}\right]_{\alpha_{2} \alpha_{3}}^{\gamma_{2}} \\
& +\left(\left[\mathbf{f}_{\mathbf{x x y} \mathbf{y}^{\prime}}\right]_{\alpha_{1} \alpha_{2} \beta_{3}}^{i}\left[\mathbf{g}_{\mathbf{x}}\right]_{\gamma_{3}}^{\beta_{3}}\left[\mathbf{h}_{\mathbf{x}}\right]_{\alpha_{3}}^{\gamma_{3}}+\left[\mathbf{f}_{\mathbf{x x y}}\right]_{\alpha_{1} \alpha_{2} \beta_{3}}^{i}\left[\mathbf{g}_{\mathbf{x}}\right]_{\alpha_{3}}^{\beta_{3}}+\left[\mathbf{f}_{\mathbf{x x x}}\right]_{\alpha_{1} \alpha_{2} \gamma_{3}}^{i}\left[\mathbf{h}_{\mathbf{x}}\right]_{\alpha_{3}}^{\gamma_{3}}+\left[\mathbf{f}_{\mathbf{x x x}}\right]_{\alpha_{1} \alpha_{2} \alpha_{3}}^{i}\right)
\end{aligned}
$$

for $i=1,2, \ldots, n$ and $\alpha_{1}, \alpha_{2}, \alpha_{3}=1,2, \ldots, n_{x}$.

\section{B.2 The expression for $\left[b^{2}\right]_{\alpha_{3}}^{i}$}

$$
\begin{aligned}
& {\left[b^{2}\right]_{\alpha_{3}}^{i}=} \\
& \left(\left[\mathbf{f}_{\mathbf{y}^{\prime} \mathbf{y}^{\prime} \mathbf{y}^{\prime}}\right]_{\beta_{1} \beta_{2} \beta_{3}}^{i}\left[\mathbf{g}_{\mathbf{x}}\right]_{\gamma_{3}}^{\beta_{3}}\left[\mathbf{h}_{\mathbf{x}}\right]_{\alpha_{3}}^{\gamma_{3}}+\left[\mathbf{f}_{\mathbf{y}^{\prime} \mathbf{y}^{\prime} \mathbf{y}}\right]_{\beta_{1} \beta_{2} \beta_{3}}^{i}\left[\mathbf{g}_{\mathbf{x}}\right]_{\alpha_{3}}^{\beta_{3}}+\left[\mathbf{f}_{\mathbf{y}^{\prime} \mathbf{y}^{\prime} \mathbf{x}^{\prime}}\right]_{\beta_{1} \beta_{2} \gamma_{3}}^{i}\left[\mathbf{h}_{\mathbf{x}}\right]_{\alpha_{3}}^{\gamma_{3}}+\left[\mathbf{f}_{\mathbf{y}^{\prime} \mathbf{y}^{\prime} \mathbf{x}}\right]_{\beta_{1} \beta_{2} \alpha_{3}}^{i}\right) \\
& \times\left[\mathbf{g}_{\mathbf{x}}\right]_{\gamma_{2}}^{\beta_{2}}[\boldsymbol{\eta}]_{\phi_{2}}^{\gamma_{2}}\left[\mathbf{g}_{\mathbf{x}}\right]_{\gamma_{1}}^{\beta_{1}}[\boldsymbol{\eta}]_{\phi_{1}}^{\gamma_{1}}[\mathbf{I}]_{\phi_{2}}^{\phi_{1}} \\
& +\left[\mathbf{f}_{\mathbf{y}^{\prime} \mathbf{y}^{\prime}}\right]_{\beta_{1} \beta_{2}}^{i}\left(\left[\mathbf{g}_{\mathbf{x x}}\right]_{\gamma_{2} \gamma_{3}}^{\beta_{2}}\left[\mathbf{h}_{\mathbf{x}}\right]_{\alpha_{3}}^{\gamma_{3}}[\boldsymbol{\eta}]_{\phi_{2}}^{\gamma_{2}}\left[\mathbf{g}_{\mathbf{x}}\right]_{\gamma_{1}}^{\beta_{1}}[\boldsymbol{\eta}]_{\phi_{1}}^{\gamma_{1}}[\mathbf{I}]_{\phi_{2}}^{\phi_{1}}+\left[\mathbf{g}_{\mathbf{x}}\right]_{\gamma_{2}}^{\beta_{2}}[\boldsymbol{\eta}]_{\phi_{2}}^{\gamma_{2}}\left[\mathbf{g}_{\mathbf{x x}}\right]_{\gamma_{1} \gamma_{3}}^{\beta_{1}}\left[\mathbf{h}_{\mathbf{x}}\right]_{\alpha_{3}}^{\gamma_{3}}[\boldsymbol{\eta}]_{\phi_{1}}^{\gamma_{1}}[\mathbf{I}]_{\phi_{2}}^{\phi_{1}}\right) \\
& +2\left(\left[\mathbf{f}_{\mathbf{y}^{\prime} \mathbf{x}^{\prime} \mathbf{y}^{\prime}}\right]_{\beta_{1} \gamma_{2} \beta_{3}}^{i}\left[\mathbf{g}_{\mathbf{x}}\right]_{\gamma_{3}}^{\beta_{3}}\left[\mathbf{h}_{\mathbf{x}}\right]_{\alpha_{3}}^{\gamma_{3}}+\left[\mathbf{f}_{\mathbf{y}^{\prime} \mathbf{x}^{\prime} \mathbf{y}}\right]_{\beta_{1} \gamma_{2} \beta_{3}}^{i}\left[\mathbf{g}_{\mathbf{x}}\right]_{\alpha_{3}}^{\beta_{3}}+\left[\mathbf{f}_{\mathbf{y}^{\prime} \mathbf{x}^{\prime} \mathbf{x}^{\prime}}\right]_{\beta_{1} \gamma_{2} \gamma_{3}}^{i}\left[\mathbf{h}_{\mathbf{x}}\right]_{\alpha_{3}}^{\gamma_{3}}+\left[\mathbf{f}_{\mathbf{y}^{\prime} \mathbf{x}^{\prime} \mathbf{x}}\right]_{\beta_{1} \gamma_{2} \alpha_{3}}^{i}\right) \\
& \times[\boldsymbol{\eta}]_{\phi_{2}}^{\gamma_{2}}\left[\mathbf{g}_{\mathbf{x}}\right]_{\gamma_{1}}^{\beta_{1}}[\boldsymbol{\eta}]_{\phi_{1}}^{\gamma_{1}}[\mathbf{I}]_{\phi_{2}}^{\phi_{1}} \\
& +2\left[\mathbf{f}_{\mathbf{y}^{\prime} \mathbf{x}^{\prime}}\right]_{\beta_{1} \gamma_{2}}^{i}[\boldsymbol{\eta}]_{\phi_{2}}^{\gamma_{2}}\left[\mathbf{g}_{\mathbf{x x}}\right]_{\gamma_{1} \gamma_{3}}^{\beta_{1}}\left[\mathbf{h}_{\mathbf{x}}\right]_{\alpha_{3}}^{\gamma_{3}}[\boldsymbol{\eta}]_{\phi_{1}}^{\gamma_{1}}[\mathbf{I}]_{\phi_{2}}^{\phi_{1}} \\
& +\left(\left[\mathbf{f}_{\mathbf{y}^{\prime} \mathbf{y}^{\prime}}\right]_{\beta_{1} \beta_{3}}^{i}\left[\mathbf{g}_{\mathbf{x}}\right]_{\gamma_{3}}^{\beta_{3}}\left[\mathbf{h}_{\mathbf{x}}\right]_{\alpha_{3}}^{\gamma_{3}}+\left[\mathbf{f}_{\mathbf{y}^{\prime} \mathbf{y}}\right]_{\beta_{1} \beta_{3}}^{i}\left[\mathbf{g}_{\mathbf{x}}\right]_{\alpha_{3}}^{\beta_{3}}+\left[\mathbf{f}_{\mathbf{y}^{\prime} \mathbf{x}^{\prime}}\right]_{\beta_{1} \gamma_{3}}^{i}\left[\mathbf{h}_{\mathbf{x}}\right]_{\alpha_{3}}^{\gamma_{3}}+\left[\mathbf{f}_{\mathbf{y}^{\prime} \mathbf{x}}\right]_{\beta_{1} \alpha_{3}}^{i}\right) \\
& \times\left(\left[\mathbf{g}_{\mathbf{x x}}\right]_{\gamma_{1} \gamma_{2}}^{\beta_{1}}[\boldsymbol{\eta}]_{\phi_{2}}^{\gamma_{2}}[\boldsymbol{\eta}]_{\phi_{1}}^{\gamma_{1}}[\mathbf{I}]_{\phi_{2}}^{\phi_{1}}+\left[\mathbf{g}_{\mathbf{x}}\right]_{\gamma_{1}}^{\beta_{1}}\left[\mathbf{h}_{\sigma \sigma}\right]^{\gamma_{1}}+\left[\mathbf{g}_{\sigma \sigma}\right]^{\beta_{1}}\right) \\
& +\left[\mathbf{f}_{\mathbf{y}^{\prime}}\right]_{\beta_{1}}^{i}\left(\left[\mathbf{g}_{\mathbf{x x x}}\right]_{\gamma_{1} \gamma_{2} \gamma_{3}}^{\beta_{1}}\left[\mathbf{h}_{\mathbf{x}}\right]_{\alpha_{3}}^{\gamma_{3}}[\boldsymbol{\eta}]_{\phi_{2}}^{\gamma_{2}}[\boldsymbol{\eta}]_{\phi_{1}}^{\gamma_{1}}[\mathbf{I}]_{\phi_{2}}^{\phi_{1}}+\left[\mathbf{g}_{\mathbf{x x}}\right]_{\gamma_{1} \gamma_{3}}^{\beta_{1}}\left[\mathbf{h}_{\mathbf{x}}\right]_{\alpha_{3}}^{\gamma_{3}}\left[\mathbf{h}_{\sigma \sigma}\right]^{\gamma_{1}}\right) \\
& +\left(\left[\mathbf{f}_{\mathbf{y y}^{\prime}}\right]_{\beta_{1} \beta_{3}}^{i}\left[\mathbf{g}_{\mathbf{x}}\right]_{\gamma_{3}}^{\beta_{3}}\left[\mathbf{h}_{\mathbf{x}}\right]_{\alpha_{3}}^{\gamma_{3}}+\left[\mathbf{f}_{\mathbf{y y}}\right]_{\beta_{1} \beta_{3}}^{i}\left[\mathbf{g}_{\mathbf{x}}\right]_{\alpha_{3}}^{\beta_{3}}+\left[\mathbf{f}_{\mathbf{y x}}\right]_{\beta_{1} \gamma_{3}}^{i}\left[\mathbf{h}_{\mathbf{x}}\right]_{\alpha_{3}}^{\gamma_{3}}+\left[\mathbf{f}_{\mathbf{y x}}\right]_{\beta_{1} \alpha_{3}}^{i}\right)\left[\mathbf{g}_{\sigma \boldsymbol{\sigma} \sigma}\right]^{\beta_{1}}
\end{aligned}
$$

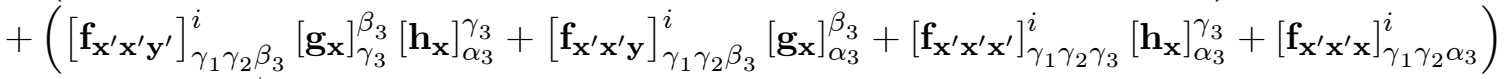

$$
\begin{aligned}
& \times[\boldsymbol{\eta}]_{\phi_{2}}^{\gamma_{2}}[\boldsymbol{\eta}]_{\phi_{1}}^{\gamma_{1}}[\mathbf{I}]_{\phi_{2}}^{\phi_{1}}
\end{aligned}
$$




$$
+\left(\left[\mathbf{f}_{\mathbf{x}^{\prime} \mathbf{y}^{\prime}}\right]_{\gamma_{1} \beta_{3}}^{i}\left[\mathbf{g}_{\mathbf{x}}\right]_{\gamma_{3}}^{\beta_{3}}\left[\mathbf{h}_{\mathbf{x}}\right]_{\alpha_{3}}^{\gamma_{3}}+\left[\mathbf{f}_{\mathbf{x}^{\prime} \mathbf{y}}\right]_{\gamma_{1} \beta_{3}}^{i}\left[\mathbf{g}_{\mathbf{x}}\right]_{\alpha_{3}}^{\beta_{3}}+\left[\mathbf{f}_{\mathbf{x}^{\prime} \mathbf{x}^{\prime}}\right]_{\gamma_{1} \gamma_{3}}^{i}\left[\mathbf{h}_{\mathbf{x}}\right]_{\alpha_{3}}^{\gamma_{3}}+\left[\mathbf{f}_{\mathbf{x}^{\prime} \mathbf{x}}\right]_{\gamma_{1} \alpha_{3}}^{i}\right)\left[\mathbf{h}_{\boldsymbol{\sigma} \boldsymbol{\sigma}}\right]^{\gamma_{1}}
$$

for $i=1,2, \ldots, n$ and $\alpha_{3}=1,2, \ldots, n_{x}$.

\section{B.3 The expression for $\left[b^{3}\right]^{i}$}

We introduce the notation that

$$
\left[\mathbf{m}^{3}\left(\boldsymbol{\epsilon}^{\prime}\right)\right]_{\phi_{2} \phi_{3}}^{\phi_{1}}=\left\{\begin{array}{cc}
m^{3}\left(\boldsymbol{\epsilon}^{\prime}\left(\phi_{1}\right)\right) & \text { if } \phi_{1}=\phi_{2}=\phi_{3} \\
0 & \text { otherwise }
\end{array}\right.
$$

where $m^{3}\left(\boldsymbol{\epsilon}^{\prime}\left(\phi_{1}\right)\right)$ denotes the third moment of $\boldsymbol{\epsilon}_{t+1}\left(\phi_{1}\right)$ for $\phi_{1}=1,2, \ldots, n_{\epsilon}$. Notice that $\mathbf{m}^{3}\left(\boldsymbol{\epsilon}^{\prime}\right)$ has dimensions $n_{\epsilon} \times n_{\epsilon} \times n_{\epsilon}$. Then:

$$
\begin{aligned}
& {\left[b^{3}\right]^{i}=\left[\mathbf{f}_{\mathbf{y}^{\prime} \mathbf{y}^{\prime} \mathbf{y}^{\prime}}\right]_{\beta_{1} \beta_{2} \beta_{3}}^{i}\left[\mathbf{g}_{\mathbf{x}}\right]_{\gamma_{3}}^{\beta_{3}}[\boldsymbol{\eta}]_{\phi_{3}}^{\gamma_{3}}\left[\mathbf{g}_{\mathbf{x}}\right]_{\gamma_{2}}^{\beta_{2}}[\boldsymbol{\eta}]_{\phi_{2}}^{\gamma_{2}}\left[\mathbf{g}_{\mathbf{x}}\right]_{\gamma_{1}}^{\beta_{1}}[\boldsymbol{\eta}]_{\phi_{1}}^{\gamma_{1}}\left[\mathbf{m}^{3}\left(\boldsymbol{\epsilon}^{\prime}\right)\right]_{\phi_{2} \phi_{3}}^{\phi_{1}}} \\
& +3\left[\mathbf{f}_{\mathbf{y}^{\prime} \mathbf{y}^{\prime} \mathbf{x}^{\prime}}\right]_{\beta_{1} \beta_{2} \gamma_{3}}^{i}[\boldsymbol{\eta}]_{\phi_{3}}^{\gamma_{3}}\left[\mathbf{g}_{\mathbf{x}}\right]_{\gamma_{2}}^{\beta_{2}}[\boldsymbol{\eta}]_{\phi_{2}}^{\gamma_{2}}\left[\mathbf{g}_{\mathbf{x}}\right]_{\gamma_{1}}^{\beta_{1}}[\boldsymbol{\eta}]_{\phi_{1}}^{\gamma_{1}}\left[\mathbf{m}^{3}\left(\boldsymbol{\epsilon}^{\prime}\right)\right]_{\phi_{2} \phi_{3}}^{\phi_{1}} \\
& +3\left[\mathbf{f}_{\mathbf{y}^{\prime} \mathbf{y}^{\prime}}\right]_{\beta_{1} \beta_{2}}^{i}\left[\mathbf{g}_{\mathbf{x x}}\right]_{\gamma_{2} \gamma_{3}}^{\beta_{2}}[\boldsymbol{\eta}]_{\phi_{3}}^{\gamma_{3}}[\boldsymbol{\eta}]_{\phi_{2}}^{\gamma_{2}}\left[\mathbf{g}_{\mathbf{x}}\right]_{\gamma_{1}}^{\beta_{1}}[\boldsymbol{\eta}]_{\phi_{1}}^{\gamma_{1}}\left[\mathbf{m}^{3}\left(\boldsymbol{\epsilon}^{\prime}\right)\right]_{\phi_{2} \phi_{3}}^{\phi_{1}} \\
& +3\left[\mathbf{f}_{\mathbf{y}^{\prime} \mathbf{x}^{\prime} \mathbf{x}^{\prime}}\right]_{\beta_{1} \gamma_{2} \gamma_{3}}^{i}[\boldsymbol{\eta}]_{\phi_{3}}^{\gamma_{3}}[\boldsymbol{\eta}]_{\phi_{2}}^{\gamma_{2}}\left[\mathbf{g}_{\mathbf{x}}\right]_{\gamma_{1}}^{\beta_{1}}[\boldsymbol{\eta}]_{\phi_{1}}^{\gamma_{1}}\left[\mathbf{m}^{3}\left(\boldsymbol{\epsilon}^{\prime}\right)\right]_{\phi_{2} \phi_{3}}^{\phi_{1}} \\
& +3\left[\mathbf{f}_{\mathbf{y}^{\prime} \mathbf{x}^{\prime}}\right]_{\beta_{1} \gamma_{2}}^{i}[\boldsymbol{\eta}]_{\phi_{2}}^{\gamma_{2}}\left[\mathbf{g}_{\mathbf{x x}}\right]_{\gamma_{1} \gamma_{3}}^{\beta_{1}}[\boldsymbol{\eta}]_{\phi_{3}}^{\gamma_{3}}[\boldsymbol{\eta}]_{\phi_{1}}^{\gamma_{1}}\left[\mathbf{m}^{3}\left(\boldsymbol{\epsilon}^{\prime}\right)\right]_{\phi_{2} \phi_{3}}^{\phi_{1}} \\
& +\left[\mathbf{f}_{\mathbf{y}^{\prime}}\right]_{\beta_{1}}^{i}\left[\mathbf{g}_{\mathbf{x x x} x}\right]_{\gamma_{1} \gamma_{2} \gamma_{3}}^{\beta_{1}}[\boldsymbol{\eta}]_{\phi_{3}}^{\gamma_{3}}[\boldsymbol{\eta}]_{\phi_{2}}^{\gamma_{2}}[\boldsymbol{\eta}]_{\phi_{1}}^{\gamma_{1}}\left[\mathbf{m}^{3}\left(\boldsymbol{\epsilon}^{\prime}\right)\right]_{\phi_{2} \phi_{3}}^{\phi_{1}} \\
& +\left[\mathbf{f}_{\mathbf{x}^{\prime} \mathbf{x}^{\prime} \mathbf{x}^{\prime}}\right]_{\gamma_{1} \gamma_{2} \gamma_{3}}^{i}[\boldsymbol{\eta}]_{\phi_{3}}^{\gamma_{3}}[\boldsymbol{\eta}]_{\phi_{2}}^{\gamma_{2}}[\boldsymbol{\eta}]_{\phi_{1}}^{\gamma_{1}}\left[\mathbf{m}^{3}\left(\boldsymbol{\epsilon}^{\prime}\right)\right]_{\phi_{2} \phi_{3}}^{\phi_{1}}
\end{aligned}
$$

\section{B.4 Matlab implementation}

Our implementation extends the one provided by Schmitt-Grohé \& Uribe (2004) for DSGE models approximated up to second order. That is, the user only needs to provide the set of equilibrium conditions in the function $\mathbf{f}\left(\mathbf{y}_{t+1}, \mathbf{y}_{t}, \mathbf{x}_{t+1}, \mathbf{x}_{t}\right)$ and values of $\mathbf{y}_{t}$ and $\mathbf{x}_{t}$ in the deterministic steady state, i.e. $\left(\mathbf{y}_{s s}, \mathbf{x}_{s s}\right)$. The function Anal_derivatives.m then computes all required analytical derivatives of $\mathbf{f}$ up to third order using the Symbolic Toolbox in Matlab. Given the steady state values $\left(\mathbf{y}_{s s}, \mathbf{x}_{s s}\right)$ and the structural coefficients, the function num_eval_3rd.m then computes the numerical values of these derivatives. The functions gx_hx.m, gxx_hxx.m, and gss_hss.m by Schmitt-Grohé \& Uribe (2004) are used to compute the first and second order derivatives of $\mathbf{g}$ and $\mathbf{h}$. All third order derivatives of $\mathbf{g}$ and $\mathbf{h}$ are finally computed by the function g_h_3rd.m.

If the user in relation to estimation or sensitivity analysis requires to solve the model many times, then it is computationally faster to print the analytical derivatives of $\mathbf{f}$ into a function and evaluate them as real matrices. That is the user can settle with only differentiating the same model once. The script Display_matlab.m is useful in this context because it allows the user to print the analytical derivatives of $\mathbf{f}$ into a text-file.

The output from for the first and second order terms are stored as in Schmitt-Grohé \& Uribe (2004). For the third order terms, the matrices $\mathbf{g}_{\mathbf{x} \mathbf{x} \mathbf{x}}$ and $\mathbf{h}_{\mathbf{x} \mathbf{x} \mathbf{x}}$ have dimensions $n_{\mathbf{y}} \times$

$n_{x} \times n_{x} \times n_{x}$ and $n_{x} \times n_{x} \times n_{x} \times n_{x}$, respectively. Here, $\mathbf{g}_{\mathbf{x x x}}\left(\beta_{1}, \alpha_{1}, \alpha_{2}, \alpha_{3}\right)=\left[\mathbf{g}_{\mathbf{x x x}}\right]_{\alpha_{1} \alpha_{2} \alpha_{3}}^{\beta_{1}}$ and $\mathbf{h}_{\mathbf{x x x}}\left(\gamma_{1}, \alpha_{1}, \alpha_{2}, \alpha_{3}\right)=\left[\mathbf{h}_{\mathbf{x x x}}\right]_{\alpha_{1} \alpha_{2} \alpha_{3}}^{\gamma_{1}}$ for $\beta_{1}=1,2, \ldots, n_{y}$ and $\gamma_{1}, \alpha_{1}, \alpha_{2}, \alpha_{3}=1,2, \ldots, n_{x}$, where the arguments for $\mathbf{g}_{\mathbf{x} \mathbf{x} \mathbf{x}}$ and $\mathbf{h}_{\mathbf{x} \mathbf{x} \mathbf{x}}$ index the elements in these matrices. Similarly, $\mathbf{g}_{\sigma \sigma \mathbf{x}}$ and 
$\mathbf{h}_{\sigma \sigma \mathbf{x}}$ have dimensions $n_{y} \times n_{x}$ and $n_{x} \times n_{x}$, respectively, and $\mathbf{g}_{\sigma \sigma \mathbf{x}}\left(\beta_{1}, \alpha_{3}\right)=\left[\mathbf{g}_{\sigma \sigma \mathbf{x}}\right]_{\alpha_{3}}^{\beta_{1}}$ and $\mathbf{h}_{\sigma \sigma \mathbf{x}}\left(\gamma_{1}, \alpha_{3}\right)=\left[\mathbf{h}_{\sigma \sigma \mathbf{x}}\right]_{\alpha_{3}}^{\gamma_{1}}$. Finally, $\mathbf{g}_{\sigma \sigma \sigma}$ and $\mathbf{h}_{\sigma \sigma \sigma}$ have dimensions $n_{y} \times 1$ and $n_{x} \times 1$, and $\mathbf{g}_{\sigma \sigma \sigma}\left(\beta_{1}, 1\right)=\left[\mathbf{g}_{\sigma \sigma \sigma}\right]^{\beta_{1}}$ and $\mathbf{h}_{\sigma \sigma \sigma}\left(\gamma_{1}, 1\right)=\left[\mathbf{h}_{\sigma \sigma \sigma}\right]^{\gamma_{1}}$. 
Table 1: Calibration

\begin{tabular}{cccc}
\hline \hline$\gamma$ & 2.5 & $\rho_{r}$ & 0.85 \\
$\nu$ & 0.35 & $n_{s s}$ & 0.38 \\
$\beta$ & 0.9995 & $\frac{g_{s s}}{y_{s s}}$ & 0.17 \\
$\alpha$ & -110 & $\pi_{s s}$ & 1.008 \\
$\theta$ & 0.36 & $\mu_{z, s s}$ & 1.005 \\
$\eta$ & 6 & $\rho_{a}$ & 0.98 \\
$\xi$ & 260 & $\rho_{g}$ & 0.90 \\
$\delta$ & 0.025 & $s t d\left(\epsilon_{a, t}\right)$ & 0.0075 \\
$\phi_{\pi}$ & 1.5 & $s t d\left(\epsilon_{g, t}\right)$ & 0.004 \\
$\phi_{y}$ & 0.3 & $s t d\left(\epsilon_{r, t}\right)$ & 0.003 \\
\hline \hline
\end{tabular}


Table 2: Technology shocks with non-symmetric innovations

Moments in the model are computed from a simulated time series of length 2,000,000 for a third order approximation to the model.

\begin{tabular}{|c|c|c|c|c|}
\hline & $1961-2007$ & Benchmark & $\begin{array}{c}\text { Non-symmetric } \\
\text { Case I }\end{array}$ & $\begin{array}{c}\text { Non-symmetric } \\
\text { Case II }\end{array}$ \\
\hline Key moments (in pct.) & (1) & $(2)$ & $(3)$ & $(4)$ \\
\hline $\operatorname{std}\left(\Delta c_{t}\right)$ & 2.69 & 3.99 & 4.02 & 3.99 \\
\hline $\operatorname{std}\left(\pi_{t}\right)$ & 2.49 & 2.23 & 2.19 & 2,22 \\
\hline $\operatorname{std}\left(r_{t, 1}\right)$ & 2.71 & 2.89 & 2.88 & 2.88 \\
\hline $\operatorname{std}\left(r_{t, 40}\right)$ & 2.41 & 1.99 & 1.98 & 1.99 \\
\hline $\operatorname{mean}\left(r_{t, 40}-r_{t}\right)$ & 1.40 & 0.97 & 1.72 & 1.04 \\
\hline $\operatorname{std}\left(r_{t, 40}-r_{t}\right)$ & 1.39 & 1.19 & 1.18 & 1.18 \\
\hline $\operatorname{mean}\left(P_{t, 40}\right)$ & 1.06 & 1.08 & 1.81 & 1.14 \\
\hline $\operatorname{std}\left(P_{t, 40}\right)$ & 0.54 & 0.02 & 0.02 & 0.02 \\
\hline \multicolumn{5}{|l|}{ Skewness } \\
\hline$\Delta c_{t}$ & -0.69 & 0.00 & -7.94 & -0.68 \\
\hline$\pi_{t}$ & 1.22 & -0.04 & 1.59 & 0.07 \\
\hline$r_{t}$ & 1.05 & -0.01 & 1.34 & 0.09 \\
\hline$r_{t, 40}$ & 0.97 & 0.00 & 1.62 & 0.11 \\
\hline \multicolumn{5}{|l|}{ Kurtosis } \\
\hline$\Delta c_{t}$ & 5.75 & 3.00 & 99.07 & 6.43 \\
\hline$\pi_{t}$ & 4.24 & 3.02 & 6.26 & 3.11 \\
\hline$r_{t}$ & 4.58 & 3.01 & 5.57 & 3.09 \\
\hline$r_{t, 40}$ & 3.60 & 3.02 & 6.42 & 3.12 \\
\hline \multicolumn{5}{|l|}{ Properties } \\
\hline $\operatorname{std}\left(a_{t}\right)$ & - & 0.0377 & 0.0377 & 0.0376 \\
\hline skew $\left(a_{t}\right)$ & - & 0.00 & -1.64 & -0.12 \\
\hline $\operatorname{kurt}\left(a_{t}\right)$ & - & 3.01 & 6.52 & 3.13 \\
\hline$\varphi$ & - & 0 & -38.67 & -7.00 \\
\hline$E\left[\epsilon_{a, t}^{3}\right]$ & - & 0 & -12.19 & -1.03 \\
\hline
\end{tabular}


Table 3: Technology shocks with stochastic volatility Moments in the model are computed from a simulated time series of length 2,000,000 for a third order approximation to the model. The calibration for the volatility process is $\rho_{\sigma_{a}}=0.99$. All the remaining parameter values are as in Table 1.

\begin{tabular}{|c|c|c|c|c|c|}
\hline & $1961-2007$ & Benchmark & $\begin{array}{c}\text { Stoch. vol. } \\
\text { Case I }\end{array}$ & $\begin{array}{c}\text { Stoch. vol. } \\
\text { Case II }\end{array}$ & $\begin{array}{c}\text { No stoch. vol. } \\
\text { High std. }\end{array}$ \\
\hline Key moments (in pct.) & (1) & (2) & $(3)$ & $(4)$ & $(5)$ \\
\hline $\operatorname{std}\left(\Delta c_{t}\right)$ & 2.69 & 3.99 & 4.00 & 4.17 & 4.25 \\
\hline $\operatorname{std}\left(\pi_{t}\right)$ & 2.49 & 2.23 & 2.25 & 2.42 & 2.42 \\
\hline$s t d\left(r_{t, 1}\right)$ & 2.71 & 2.89 & 2.92 & 3.11 & 3.11 \\
\hline $\operatorname{std}\left(r_{t, 40}\right)$ & 2.41 & 1.99 & 2.02 & 2.18 & 2.16 \\
\hline mean $\left(r_{t, 40}-r_{t}\right)$ & 1.40 & 0.97 & 0.97 & 0.96 & 1.14 \\
\hline $\operatorname{std}\left(r_{t, 40}-r_{t}\right)$ & 1.39 & 1.19 & 1.20 & 1.27 & 1.23 \\
\hline $\operatorname{mean}\left(P_{t, 40}\right)$ & 1.06 & 1.08 & 1.08 & 1.08 & 1.27 \\
\hline $\operatorname{std}\left(P_{t, 40}\right)$ & 0.54 & 0.02 & 0.14 & 0.40 & 0.03 \\
\hline \multicolumn{6}{|l|}{ Skewness } \\
\hline$\Delta c_{t}$ & -0.69 & 0.00 & 0.00 & 0.00 & 0.00 \\
\hline$\pi_{t}$ & 1.22 & -0.04 & -0.03 & -0.02 & -0.05 \\
\hline$r_{t}$ & 1.05 & -0.01 & -0.01 & -0.10 & -0.01 \\
\hline$r_{t, 40}$ & 0.97 & 0.00 & 0.02 & 0.12 & 0.00 \\
\hline \multicolumn{6}{|l|}{ Kurtosis } \\
\hline$\Delta c_{t}$ & 5.75 & 3.00 & 3.04 & 3.34 & 3.00 \\
\hline$\pi_{t}$ & 4.24 & 3.02 & 3.10 & 3.92 & 3.02 \\
\hline$r_{t}$ & 4.58 & 3.01 & 3.08 & 3.79 & 3.01 \\
\hline$r_{t, 40}$ & 3.60 & 3.02 & 3.10 & 3.92 & 3.02 \\
\hline \multicolumn{6}{|l|}{ Properties } \\
\hline $\operatorname{std}\left(a_{t}\right)$ & - & 0.0377 & 0.0382 & 0.0409 & 0.0410 \\
\hline $\operatorname{std}\left(\sigma_{a, t}\right)$ & - & 0 & 0.071 & 0.214 & 0 \\
\hline skew $\left(a_{t}\right)$ & - & 0.00 & -0.01 & -0.03 & -0.01 \\
\hline $\operatorname{kurt}\left(a_{t}\right)$ & - & 3.01 & 3.10 & 3.93 & 3.01 \\
\hline $\operatorname{std}\left(\epsilon_{\sigma_{a}, t}\right)$ & - & 0.00 & 0.01 & 0.03 & 0.00 \\
\hline
\end{tabular}


Table 4: Market price of risk and quantity of risk

The moments are computed from a simulated time series of length 2,000,000 for a third order approximation to the model. The term premium is not expressed in annual basis points for this decomposition.

\begin{tabular}{l|c|cc|cc}
\hline \hline & Benchmark & $\begin{array}{c}\text { Stoch. vol. } \\
\text { Case I }\end{array}$ & $\begin{array}{c}\text { Stoch. vol. } \\
\text { Case II }\end{array}$ & $\begin{array}{c}\text { GARCH } \\
\text { Case I }\end{array}$ & $\begin{array}{c}\text { GARCH } \\
\text { Case II }\end{array}$ \\
\hline Mean & 0.017 & 0.017 & 0.017 & 0.021 & 0.042 \\
$\operatorname{Var}_{t}\left(M_{t, t+1}\right)$ & 0.017 & 0.017 & 0.017 & 0.021 & 0.043 \\
Market price of risk & 0.157 & 0.158 & 0.165 & 0.145 & 0.117 \\
Quantity of risk & & & & & \\
Standard deviation & & & & & \\
$\operatorname{Var}_{t}\left(M_{t, t+1}\right)$ & 0.0008 & 0.0025 & 0.0072 & 0.0012 & 0.0061 \\
Market price of risk & 0.0009 & 0.0026 & 0.0073 & 0.0013 & 0.0062 \\
Quantity of risk & 0.0049 & 0.0060 & 0.3302 & 0.0044 & 0.0042 \\
\hline \hline
\end{tabular}


Table 5: Technologi shocks with GARCH

Moments in the model are computed from a simulated time series of length 2,000,000 for a third order approximation to the model. The value of $\rho_{1}$ is 0.95 .

\begin{tabular}{|c|c|c|c|c|c|}
\hline & $1961-2007$ & Benchmark & $\begin{array}{c}\text { GARCH } \\
\text { Case I }\end{array}$ & $\begin{array}{c}\text { GARCH } \\
\text { Case II }\end{array}$ & $\begin{array}{c}\text { No GARCH } \\
\text { High std. }\end{array}$ \\
\hline Key moments (in pct.) & (1) & (2) & $(3)$ & $(4)$ & $(5)$ \\
\hline $\operatorname{std}\left(\Delta c_{t}\right)$ & 2.69 & 3.98 & 4.35 & 6.53 & 4.49 \\
\hline $\operatorname{std}\left(\pi_{t}\right)$ & 2.49 & 2.23 & 2.48 & 2.60 & 2.58 \\
\hline$s t d\left(r_{t, 1}\right)$ & 2.71 & 2.89 & 3.19 & 3.26 & 3.30 \\
\hline$s t d\left(r_{t, 40}\right)$ & 2.41 & 1.99 & 2.22 & 2.30 & 2.31 \\
\hline mean $\left(r_{t, 40}-r_{t}\right)$ & 1.40 & 0.97 & 1.15 & 2.16 & 1.30 \\
\hline $\operatorname{std}\left(r_{t, 40}-r_{t}\right)$ & 1.39 & 1.19 & 1.25 & 1.33 & 1.27 \\
\hline $\operatorname{mean}\left(P_{t, 40}\right)$ & 1.06 & 1.08 & 1.22 & 1.99 & 1.45 \\
\hline $\operatorname{std}\left(P_{t, 40}\right)$ & 0.54 & 0.02 & 0.04 & 0.22 & 0.03 \\
\hline \multicolumn{6}{|l|}{ Skewness } \\
\hline$\Delta c_{t}$ & -0.69 & 0.00 & -0.00 & 0.01 & 0.00 \\
\hline$\pi_{t}$ & 1.22 & -0.04 & -0.04 & -0.04 & -0.05 \\
\hline$r_{t}$ & 1.05 & -0.01 & -0.01 & -0.01 & -0.01 \\
\hline$r_{t, 40}$ & 0.97 & -0.00 & 0.00 & 0.00 & -0.01 \\
\hline \multicolumn{6}{|l|}{ Kurtosis } \\
\hline$\Delta c_{t}$ & 5.75 & 3.00 & 3.01 & 3.14 & 3.00 \\
\hline$\pi_{t}$ & 4.24 & 3.02 & 3.04 & 2.98 & 3.02 \\
\hline$r_{t}$ & 4.58 & 3.01 & 3.03 & 2.96 & 3.01 \\
\hline$r_{t, 40}$ & 3.60 & 3.02 & 3.04 & 2.97 & 3.02 \\
\hline \multicolumn{6}{|l|}{ Properties } \\
\hline $\operatorname{std}\left(a_{t}\right)$ & - & 0.0377 & 0.0420 & 0.0437 & 0.0438 \\
\hline $\operatorname{std}\left(\sigma_{a, t}\right)$ & - & 0 & 0.0283 & 0.190 & 0 \\
\hline skew $\left(a_{t}\right)$ & - & 0.00 & -0.01 & 0.00 & -0.01 \\
\hline kurt $\left(a_{t}\right)$ & - & 3.01 & 3.04 & 2.97 & 3.01 \\
\hline$\rho_{2}$ & - & 0.00 & 0.01 & 0.04 & 0.00 \\
\hline
\end{tabular}




\section{References}

Andreasen, M. M. (2010), 'Stochastic volatility and DSGE models', Economics Letters 108, 7-9.

Arouba, S. B., Fernández-Villaverde, J. \& Rubio-Ramírez, J. F. (2005), 'Comparing solution methods for dynamic equilibrium economies', Journal of Economic Dynamics and Control 20(2), 891-910.

Aruoba, S. B., Fernandez-Villaverde, J. \& Rubio-Ramirez, J. F. (2006), 'Comparing solution methods for dynamic equilibrium economies', Journal of Economic Dynamics and Control 30, 2477-2508.

Backus, D. K., Gregory, A. W. \& Zin, S. E. (1989), 'Risk premiums in the term structure: Evidence from artificial economics', Journal of Monetary Economics 24, 371-399.

Bansal, R. \& Yaron, A. (2004), 'Risks for the long run: A potential resolution of asset pricing puzzles', The Journal of Finance 59(4), 1481-1509.

Barro, R. J. (2006), 'Rare disasters and asset markets in the twentieth century', The Quarterly Journal of Economics pp. 823-866.

Barro, R. J. (2009), 'Rare disasters, asset prices, and welfare costs', American Economic Review 99(1), 243-264.

Bekaert, G., Cho, S. \& Moreno, A. (2010), 'New keynesian macroeconomics and the term structure', Journal of Money, Credit and Banking 42(1), 33-62.

Binsbergen, J. H. V., Fernandez-Villaverde, J., Koijen, R. S. \& Rubio-Ramirez, J. (2010), 'The term structure of interest rates in a DSGE model with recursive preferences', PIER Working Paper 10-011.

Bloom, N. (2009), 'The impact of uncertainty shocks', Econometrica 77(3), 623-685.

Boldrin, M., Christiano, L. J. \& Fisher, J. D. M. (2001), 'Habit persistence: Asset returns, and the business cycle', The American Economic Review 91(1), 149-166.

Bollerslev, T. (1986), 'Generalized autoregressive conditional heteroskedasticity', Journal of Econometrics 31, 307-327.

Campbell, J. Y. \& Cochrane, J. H. (1999), 'By force of habit: A consumption-based explanation of aggregate stock market behavior', Journal of Political Economy 107(2), 205-251.

Campbell, J. Y. \& Shiller, R. J. (1991), 'Yield spread and interest rate movements: A bird's eye view', The Review of Economic Studies 58(3), 495-514.

Chari, V. V., Kehoe, P. J. \& McGrattan, E. R. (2002), 'Can sticky price models generate volatile and persistent real exchange rates?', Review of Economic Studies 69, 533-563.

Cochrane, J. H. (2001), 'Asset pricing', Princeton University Press .

Cox, J. C., Ingersoll, J. E. \& Ross, S. A. (1985), 'A theory of the term structure of interest rates', Econometrica 53(2), 385-407. 
Dai, Q. \& Singleton, K. J. (2002), 'Expectation puzzles, time-varying risk premia and affine models of the term structure', Journal of Financial Economics 63, 415-441.

De Paoli, B., Scott, A. \& Weeken, O. (2007), 'Asset pricing implications of a new keynesian model', Bank of England Working Paper no. 326 .

Epstein, L. G. \& Zin, S. E. (1989), 'Substitution, risk aversion, and the temporal behavior of consumption and asset returns: A theoretical framework', Econometrica 57(4), 937-969.

Fernández-Villaverde, J., Guerrón-Quintana, P., Rubio-Ramírez, J. F. \& Uribe, M. (2010), 'Risk matters: The real effects of volatility shocks', Working Paper.

Fernández-Villaverde, J. \& Rubio-Ramírez, J. F. (2007), 'Estimating macroeconomic models: A likelihood approach', Review of Economic Studies pp. 1-46.

Gabaix, X. (2008), 'Variable rare disasters: A tractable theory of ten puzzles in macro-finance', American Economic Review: Papers and Proceedings 2, 64-67.

Galí, J. (2008), 'Monetary policy, inflation, and the business cycle: An introduction to the new keynesian framework', Princeton University Press .

Gürkaynak, R., Sack, B. \& Wright, J. (2007), 'The u.s. treasure yield curve: 1961 to the present', Journal of Monetary Economics 54, 2291-2304.

Hordahl, P., Tristani, O. \& Vestin, D. (2008), 'The yield curve and macroeconomic dynamics', The Economic Journal 118, 1937-1970.

Jermann, U. J. (1998), 'Asset pricing in production economics', Journal of Monetary Economics 41, 257-275.

Judd, K. L. \& Guu, S.-M. (1997), 'Asymptotic methods for aggregate growth models', Journal of Economic Dynamics and Control 21, 1025-1042.

Justiniano, A. \& Primiceri, G. E. (2008), 'The time-varying volatility of macroeconomic fluctuations', The American Economic Review pp. 604-641.

Keen, B. \& Wang, Y. (2007), 'What is a realistic value for price adjustment costs in new keynesian models?', Applied Economics Letters 14, 789-793.

Lucas, R. E. (1978), 'Asset prices in an exchange economy', Econometrica 46(6), 1429-1445.

Mark, N. C. (1985), 'On time varying risk premia in the foreign exchange market', Journal of Monetary Economics 16, 3-18.

Mehra, R. \& Prescott, E. C. (1985), 'The equity premium: A puzzle', Journal of Monetary Economics 15, 145-161.

Rietz, T. A. (1988), 'The equity premium: A solution', Journal of Monetary Economics 22, 117131.

Rotemberg, J. J. (1982), 'Monopolistic price adjustment and aggregate output', The Review of Economic Studies 49, 517-531. 
Rudebusch, G. D. \& Swanson, E. T. (2009), 'The bond premium in a DSGE model with long-run real and nominal risks', Working Paper .

Schmitt-Grohé, S. \& Uribe, M. (2004), 'Solving dynamic general equilibrium models using a second-order approximation to the policy function', Journal of Economic Dynamics and Control 28, 755-775.

Swanson, E. (2010), 'Risk aversion and the labor margin in dynamic equilibrium models', Working Paper.

Swanson, E., Anderson, G. \& Levin, A. (2005), 'Higher-order perturbation solutions to dynamic, discrete-time rational expectations models', Working Paper .

Tsionas, E. G. (2003), 'Exact solution of asset pricing models with arbitrary shock distributions', Journal of Economic Dynamic and Control 27, 843-851.

Uhlig, H. (2007), 'Explaining asset prices with external habits and wage rigidities', The American Economic Review: Papers and Proceedings 97(2), 239-243.

Weil, P. (1990), 'Nonexpected utility in macroeconomics', Quarterly Journal of Economics 105(1), 29-42.

Wu, T. (2006), 'Macro factors and the affine term structure of interest rates', Journal of Money, Credit, and Banking 30, 1847-1875. 
Figure 1: Impulse responses to a volatility shock in technology

Impulse responses for a one standard deviation shock to the conditional volatility in technology when the model is approximated up to third order. The effects for the two interest rates, the 10-year term premium, and consumption are expressed in annualized percentage deviation from the steady state.

The level of technology and the volatility in technology are expressed in percentage deviation from the steady state. The calibration for the volatility process is $\rho_{\sigma_{a}}=0.99$ and $\sqrt{\operatorname{Var}\left(\epsilon_{\sigma, t}\right)}=0.03^{2}$. All the remaining parameter values are as stated in Table 1.
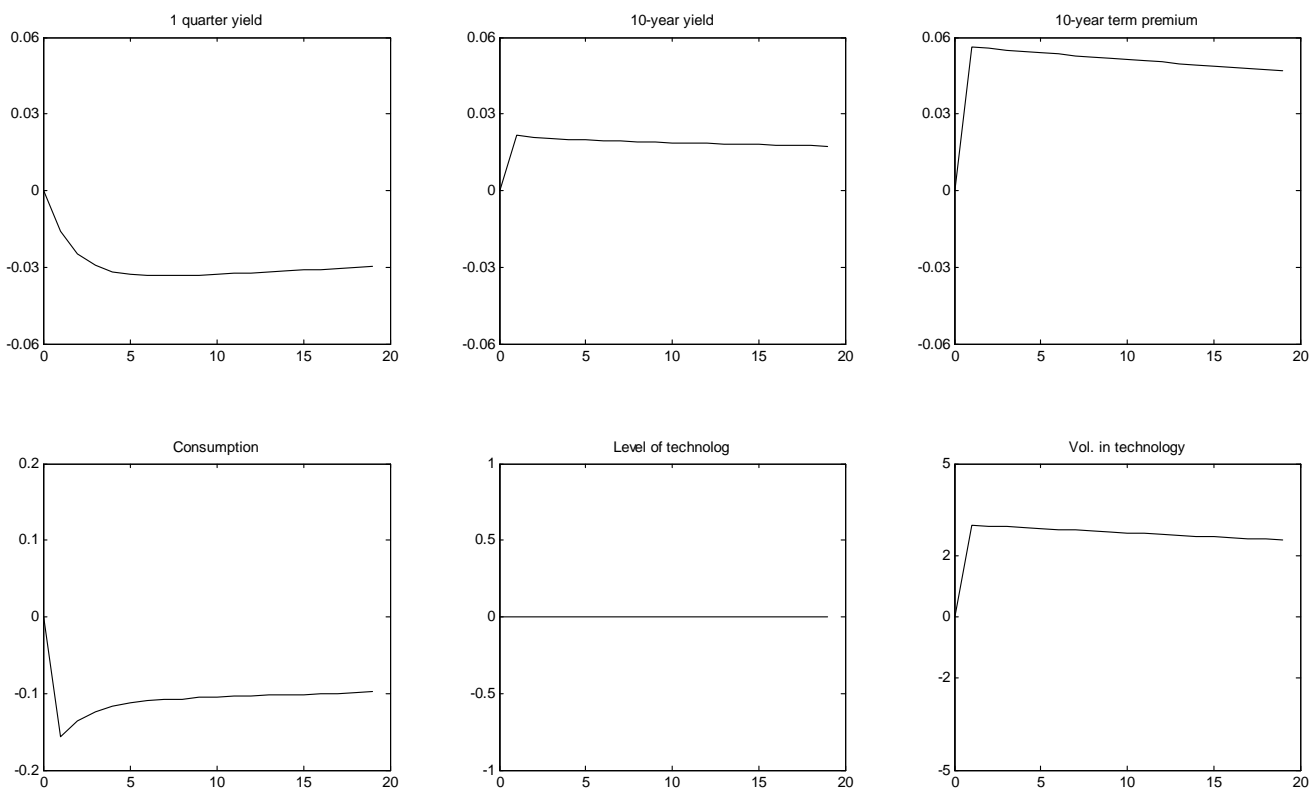
Figure 2: Impulse responses to a technology shock with GARCH

Impulse responses for a one standard deviation shock to technology when the model is approximated up to third order. The effects for the two interest rates, the 10-year term premium, and consumption are expressed in annualized percentage deviation from the steady state. The level of technology and the volatility in technology are expressed in percentage deviation from the steady state. The calibration for the GARCH process is $\rho_{1}=0.95$ and $\rho_{2}=0.04$. All the remaining parameter values are as stated in Table 1.
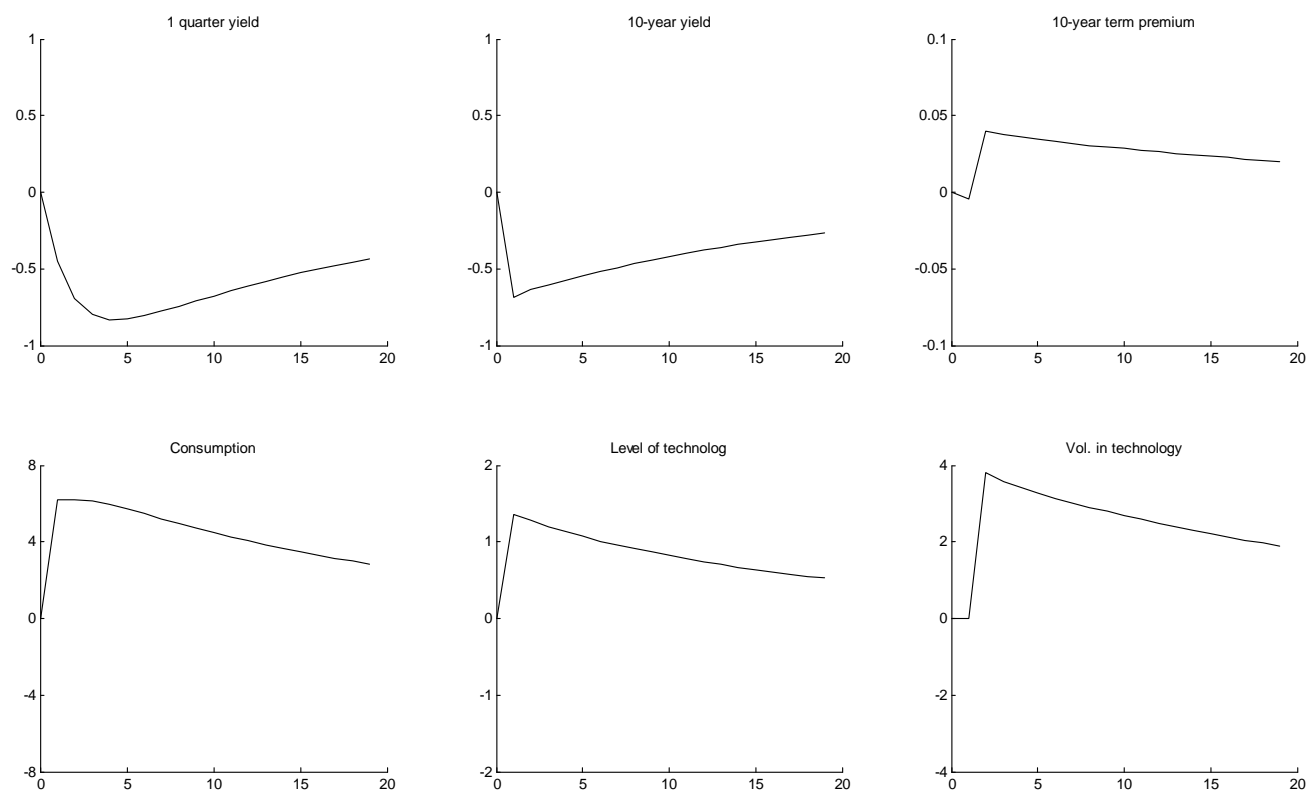
2010-49: $\quad$ Christian D. Dick, Maik Schmeling and Andreas Schrimpf: Macro Expectations, Aggregate Uncertainty, and Expected Term Premia

2010-50: Bent J esper Christensen and Petra Posedel: The Risk-Return Tradeoff and Leverage Effect in a Stochastic Volatility-in-Mean Model

2010-51: $\quad$ Christos Ntantamis: A Duration Hidden Markov Model for the Identification of Regimes in Stock Market Returns

2010-52: Christos Ntantamis: Detecting Structural Breaks using Hidden Markov Models

2010-53: Christos Ntantamis: Detecting Housing Submarkets using Unsupervised Learning of Finite Mixture Models

2010-54: Stefan Holst Bache: Minimax Regression Quantiles

2010-55: Nektarios Aslanidis and Charlotte Christiansen: Sign and Quantiles of the Realized Stock-Bond Correlation

2010-56: $\quad$ Anders Bredahl Kock: Oracle Efficient Variable Selection in Random and Fixed Effects Panel Data Models

2010-57: Charlotte Christiansen, J uanna Schröter J oensen and J esper Rangvid: The Effects of Marriage and Divorce on Financial Investments: Learning to Love or Hate Risk?

2010-58: Charlotte Christiansen, Maik Schmeling and Andreas Schrimpf: A Comprehensive Look at Financial Volatility Prediction by Economic Variables

2010-59: J ames G. MacKinnon and Morten Ørregaard Nielsen: Numerical distribution functions of fractional unit root and cointegration tests

2010-60: Bent J esper Christensen and Paolo Santucci de Magistris: Level Shifts in Volatility and the Implied-Realized Volatility Relation

2010-61: Christian Bach and Bent J esper Christensen: Latent Integrated Stochastic Volatility, Realized Volatility, and Implied Volatility: A State Space Approach

2010-62: Bent J esper Christensen and Malene Kallestrup Lamb: The Impact of Health Changes on Labor Supply: Evidence from Merged Data on Individual Objective Medical Diagnosis Codes and Early Retirement Behavior

2010-63: Martin M. Andreasen: How Non-Gaussian Shocks Affect Risk Premia in Non-Linear DSGE Models 